\title{
P-Chiral o-Phosphinophenol as a P/O Hybrid Ligand: Preparation and Use in Cu- Catalyzed Asymmetric Conjugate Addition of Diethylzinc to Acyclic Enones
}

\author{
Yukitoshi Takahashi, ${ }^{\dagger}$ Yoshikazu Yamamoto, ${ }^{\dagger}$ Kosuke Katagiri, ${ }^{\dagger \ddagger}$ Hiroshi Danjo, ${ }^{\ddagger}$ Kentaro \\ Yamaguchi, ${ }^{\ddagger}$ and Tsuneo Imamoto*, ${ }^{*}$ \\ ${ }^{\dagger}$ Department of Chemistry, Faculty of Science, Chiba University, 1-33 Yayoi-cho, Inage-ku, Chiba \\ 263-8522, Japan \\ ${ }^{\ddagger}$ Department of Pharmaceutical Technology, Faculty of Pharmaceutical Science at Kagawa Campus, \\ Tokushima Bunri University, 1314-1 Shido, Sanuki, Kagawa 769-2193, Japan \\ imamoto@faculty.chiba-u.jp
}

\section{LIST OF CONTENT}

General $\ldots \ldots \ldots$

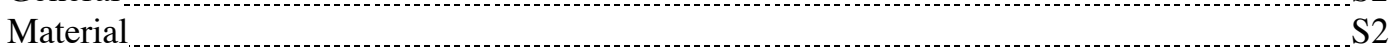

Conversion of $\mathbf{2 a}$ to $\mathbf{2 b}$ : Determination of absolute configuration of $\mathbf{2 a} \quad \mathrm{S} 3$

Analytical and spectral characterization data of epi-1a, 3b, 7 and 1,4-adducts __....... S3-S8

NMR spectra of 1a, epi-1a, 2a, 3a, 3b and 7

$\mathrm{X}$-ray structure of $\mathbf{2 b} \quad$ S26 
General. All manipulations were carried out under argon or nitrogen atmosphere. NMR spectra were recorded on a JEOL JMN-LA500 spectrometer $\left(500 \mathrm{MHz}\right.$ for ${ }^{1} \mathrm{H}, 202 \mathrm{MHz}$ for ${ }^{31} \mathrm{P}$, and 125 $\mathrm{MHz}$ for ${ }^{13} \mathrm{C}$ ) or a JEOL JMN-LA400 spectrometer (400 MHz for ${ }^{1} \mathrm{H}$ and $100 \mathrm{MHz}$ for ${ }^{13} \mathrm{C}$ ). Chemical shifts are reported in $\mathrm{d}$ ppm referenced to an internal tetramethylsilane standard for ${ }^{1} \mathrm{H}$ NMR, and to an external $85 \% \mathrm{H}_{3} \mathrm{PO}_{4}$ standard for ${ }^{31} \mathrm{P}$ NMR. Residual chloroform $\left(\delta 77.0\right.$ for $\left.{ }^{13} \mathrm{C}\right)$ was used as internal reference for ${ }^{13} \mathrm{C}$ NMR. ${ }^{1} \mathrm{H},{ }^{13} \mathrm{C}$, and ${ }^{31} \mathrm{P}$ NMR spectra were recorded in $\mathrm{CDCl}_{3}$ at $25{ }^{\circ} \mathrm{C}$ unless otherwise noted. IR spectra were recorded on a Jasco FT/IR-200. Optical rotations were recorded on a Jasco DIP-1020 polarimeter with a sodium lamp. MS (FAB and EI) spectra were recorded on a JEOL HX-110 and a JEOL AX-500 spectrometers. HPLC analyses were performed on a Shimadzu LC-10AD pump and a SPD-10A UV detector with a chiral column. GLC analyses were performed on a Shimadzu GC-17A instrument equipped with a flame ionization detector and a capillary column.

Materials. All reagents were obtained from commercial sources and used without further purification. All solvents were freshly distilled. $\mathbf{3 b}, \mathbf{4}$ and enones are known compounds. 
Conversion of 2a to $2 \mathrm{~b}$ : determination of absolute configuration of $2 \mathrm{a}$.

To a $1 \mathrm{~mL}$ THF solution of a phosphine-borane 2a $(0.27 \mathrm{mmol}, 69 \mathrm{mg})$ was added tetrafluoroboric acid $(4.0 \mathrm{mmol}, 0.55 \mathrm{~mL})$ in diethyl ether slowly at $-40{ }^{\circ} \mathrm{C}$ under nitrogen atmosphere. After stirring at room temperature for $13 \mathrm{~h}$, degassed saturated $\mathrm{NaHCO}_{3}$ was added to the solution at $0{ }^{\circ} \mathrm{C}$, and stirred for $1 \mathrm{~h}$ at room temperature. The organic layer was separated, and the aqueous layer was extracted with degassed diethyl ether five times. The combined extract was dried over $\mathrm{Na}_{2} \mathrm{SO}_{4}$ under nitrogen. The solution was passed through a column of silica gel with degassed diethyl ether elution. The eluent was concentrated under reduced pressure to give free phosphine $\mathbf{3 a}$ (40 $\mathrm{mg}, 75 \%$ yield).

Borane-THF complex (1 mmol, $1 \mathrm{~mL}$ of $1.0 \mathrm{M}$ THF solution) was added to a solution of $3 \mathbf{a}$ ( $24 \mathrm{mg}$, $0.12 \mathrm{mmol})$ in THF $(6 \mathrm{~mL})$ at $0{ }^{\circ} \mathrm{C}$ under nitrogen atmosphere. After stirring at room temperature for $1 \mathrm{~h}$, the reaction mixture was quenched by ice water containing $\mathrm{HCl}$ and diluted with diethyl ether. The organic layer was separated, and the aqueous layer was extracted with diethyl ether. The combined extract was washed with saturated $\mathrm{NaHCO}_{3}$ and brine, and dried over $\mathrm{Na}_{2} \mathrm{SO}_{4}$. After filtration, the filtrate was concentrated under reduced pressure. The residue was purified by column chromatography on silica gel (hexane/ethyl acetate $=5 / 1$ ) to give tert-butyl(2hydroxyphenyl)methylphosphine-borane 7 (26 $\mathrm{mg}, 99 \%$ yield).

Methyl iodide $(0.36 \mathrm{mmol}, 23 \mu \mathrm{L})$ was added to a solution of $7(0.12 \mathrm{mmol}, 26.2 \mathrm{mg})$ and potassium carbonate $(0.36 \mathrm{mmol}, 50 \mathrm{mg})$ in acetone at room temperature under nitrogen atmosphere. After stirring for $30 \mathrm{~h}$, the reaction mixture was concentrated under reduced pressure, and the residue was purified by column chromatography on silica gel (hexane/ethyl acetate $=5 / 1)$ to give $\mathbf{2 b}(23.2 \mathrm{mg}$, $86 \%$ yield $)$. $[\alpha]_{\mathrm{D}}{ }^{23}=+50.8^{\circ}\left(c=1.00, \mathrm{CHCl}_{3}\right)$. The absolute configuration of $2 \mathrm{a}$ was determined to be $S_{\mathrm{P}}$ by comparison of the sign of optical rotation. The enantiomeric excess was determined to be $97 \%$ by HPLC (Chiralcel OD-H, hexane $/ 2$-propanol $=99 / 1,0.5 \mathrm{~mL} / \mathrm{min}, 254 \mathrm{~nm}$ ). Retention time: $9.8 \min [(R)$-enantiomer $], 10.2 \min [(S)$-enantiomer $]$.

(R)-tert-Butyl((1R,2S,5R)-2-isopropyl-5-methylcyclohexyloxy)(2(methoxy)methoxyphenyl)phosphine-borane (epi-1a). ${ }^{1} \mathrm{H}$ NMR $\left(500 \mathrm{MHz}, \mathrm{CDCl}_{3}\right) \delta 7.69$ (ddd, $J=1.7,7.7,9.7 \mathrm{~Hz}, 1 \mathrm{H}), 7.42$ (dddd, $J=0.9,1.7,7.3,8.4 \mathrm{~Hz}, 1 \mathrm{H}$ ), 7.17 (ddd, $J=0.6,4.3,8.4,1 \mathrm{H}$ ), 7.05 (dddd, $J=0.6,0.8,7.3,7.7 \mathrm{~Hz}, 1 \mathrm{H}), 5.19(\mathrm{~d}, J=7.0 \mathrm{~Hz}, 1 \mathrm{H}), 5.15(\mathrm{~d}, J=6.8 \mathrm{~Hz}, 1 \mathrm{H}), 4.32-$ $4.25(\mathrm{~m}, 1 \mathrm{H}), 3.52(\mathrm{~s}, 3 \mathrm{H}), 2.37-2.30(\mathrm{~m}, 1 \mathrm{H}), 1.88-1.82(\mathrm{~m}, 1 \mathrm{H}), 1.70-1.58(\mathrm{~m}, 2 \mathrm{H}), 1.50-1.43(\mathrm{~m}$, $1 \mathrm{H}), 1.40-1.29(\mathrm{~m}, 1 \mathrm{H}), 1.20-0.70(\mathrm{~m}, 21 \mathrm{H}) ;{ }^{13} \mathrm{C} \mathrm{NMR}\left(125 \mathrm{MHz}, \mathrm{CDCl}_{3}\right) \delta 159.3(\mathrm{~d}, J=6.1 \mathrm{~Hz})$, $134.2(\mathrm{~d}, J=6.0 \mathrm{~Hz}), 132.9(\mathrm{~d}, J=2.0 \mathrm{~Hz}), 120.8(\mathrm{~d}, J=8.0 \mathrm{~Hz}), 120.6(\mathrm{~d}, J=28.0 \mathrm{~Hz}), 144.6(\mathrm{~d}, J$ $=6.0 \mathrm{~Hz}), 94.8,79.1(\mathrm{~d}, J=5.0 \mathrm{~Hz}), 56.4,49.4(\mathrm{~d}, J=7.0 \mathrm{~Hz}), 42.9,34.2(\mathrm{~d}, J=28.0 \mathrm{~Hz}), 33.7$, $31.4(\mathrm{~d}, J=33.0 \mathrm{~Hz}), 25.3,24.6(\mathrm{~d}, J=3.0 \mathrm{~Hz}), 22.6,22.0,21.1,15.4,14.0 ;{ }^{31} \mathrm{P}$ NMR $(202 \mathrm{MHz}$, $\mathrm{CDCl}_{3}$ ) $\delta 115.4(\mathrm{~s}) ; \mathrm{IR}$ (NaCl plates) 2960, 2385, 1590, 1485, 1155, 1085, $985 \mathrm{~cm}^{-1} ;[\alpha]_{\mathrm{D}}^{22}=+13.3^{\circ}$ $\left(c=1.07, \mathrm{CHCl}_{3}\right)$; HRMS Calcd for $\mathrm{C}_{22} \mathrm{H}_{40} \mathrm{BKO}_{3} \mathrm{P}[\mathrm{M}+\mathrm{K}]^{+}$433.2445, Found 433.2412. Anal. Calcd for $\mathrm{C}_{22} \mathrm{H}_{40} \mathrm{BO}_{3} \mathrm{P}: \mathrm{C}, 67.01 ; \mathrm{H}, 10.22$. Found: $\mathrm{C}, 66.93 ; \mathrm{H}, 10.21$.

(S)-tert-Butyl(2-methoxyphenyl)methylphosphine (3b). ${ }^{1} \mathrm{H}$ NMR (400 MHz, $\left.\mathrm{CDCl}_{3}\right) \delta$ 7.34-7.28 $(\mathrm{m}, 2 \mathrm{H}), 6.99-6.95(\mathrm{~m}, 1 \mathrm{H}), 6.89-6.85(\mathrm{~m}, 1 \mathrm{H}), 3.83(\mathrm{~s}, 3 \mathrm{H}), 1.25(\mathrm{~d}, J=5.2 \mathrm{~Hz}, 3 \mathrm{H}), 0.99(\mathrm{~d}, J=$ $11.9 \mathrm{~Hz}, 9 \mathrm{H}) ;{ }^{13} \mathrm{C}$ NMR $\left(125 \mathrm{MHz}, \mathrm{CDCl}_{3}\right) \delta 162.4(\mathrm{~d}, J=15.0 \mathrm{~Hz}), 132.5(\mathrm{~d}, J=25.0 \mathrm{~Hz}), 130.0$, $125.2(\mathrm{~d}, J=20.0 \mathrm{~Hz}), 120.3,110.2(\mathrm{~d}, J=2.0 \mathrm{~Hz}), 55.2,29.4(\mathrm{~d}, J=12.0 \mathrm{~Hz}), 27.2(\mathrm{~d}, J=14.0$ $\mathrm{Hz}), 5.1(\mathrm{~d}, J=18.1 \mathrm{~Hz}) ;{ }^{31} \mathrm{P}$ NMR $\left(202 \mathrm{MHz}, \mathrm{CDCl}_{3}\right) \delta-27.4(\mathrm{~s})$; HRMS Calcd for $\mathrm{C}_{12} \mathrm{H}_{20} \mathrm{OP}$ $[\mathrm{M}+\mathrm{H}]^{+}$211.1252, Found 211.1247.

(S)-tert-Butyl(2-hydroxyphenyl)methylphosphine-borane (7). ${ }^{1} \mathrm{H} \mathrm{NMR}\left(500 \mathrm{MHz}, \mathrm{CDCl}_{3}\right) \delta 7.85$ $(\mathrm{s}, 1 \mathrm{H}), 7.43-7.39(\mathrm{~m}, 1 \mathrm{H}), 7.26-7.23(\mathrm{~m}, 1 \mathrm{H}), 6.98-6.94(\mathrm{~m}, 2 \mathrm{H}), 1.59(\mathrm{~d}, J=9.9 \mathrm{~Hz}, 3 \mathrm{H}), 1.40-0.54$ $(\mathrm{m}, 3 \mathrm{H}), 1.15(\mathrm{~d}, J=14.7 \mathrm{~Hz}, 9 \mathrm{H}) ;{ }^{31} \mathrm{P}$ NMR $\left(202 \mathrm{MHz}, \mathrm{CDCl}_{3}\right) \delta 17.4(\mathrm{q}, J=55.0 \mathrm{~Hz}) ; \mathrm{IR}(\mathrm{NaCl}$ plates) $3405,2965,2360,1595,1435,1340,1290,1200,1080,890,760 \mathrm{~cm}^{-1}$. Anal. Calcd for $\mathrm{C}_{11} \mathrm{H}_{20}$ BOP: C, 62.90; H, 9.60. Found: C, 62.80; H, 9.38.

1,3-Diphenylpentan-1-one. ${ }^{1} \mathrm{H}$ NMR $\left(400 \mathrm{MHz}, \mathrm{CDCl}_{3}\right) \delta$ 7.92-7.87 (m, 2H), 7.55-7.50 (m, $\left.1 \mathrm{H}\right)$, 7.45-7.40 (m, 2H), 7.31-7.15 (m, 5H), 3.31-3.22 (m, 3H), 1.85-1.74 (m, 1H), 1.70-1.57 (m, 1H), 0.80 
(t, $J=7.4 \mathrm{~Hz}, 3 \mathrm{H}) ;{ }^{13} \mathrm{C}$ NMR $\left(100 \mathrm{MHz}, \mathrm{CDCl}_{3}\right) \delta$ 199.2, 144.6, 137.2, 132.9, 128.5, 128.4128 .0 , 127.6, 126.2, 45.6 43.0, 29.2, 12.1; IR (NaCl plates) $1685 \mathrm{~cm}^{-1}$; HRMS calcd for $\mathrm{C}_{17} \mathrm{H}_{19} \mathrm{O}[\mathrm{M}+\mathrm{H}]^{+}$ 139.1439, found 239.1434; The enantiomeric excess was determined by HPLC (Chiralcel OD-H, hexane $/ 2$-propanol $=199 / 1,0.5 \mathrm{~mL} / \mathrm{min}, 254 \mathrm{~nm})$. Retention time: $23.7 \mathrm{~min}[(S)$-enantiomer], 25.8 $\min [(R)$-enantiomer].

3-(4-Methoxyphenyl)-1-phenylpentan-1-one. ${ }^{1} \mathrm{H}$ NMR $\left(400 \mathrm{MHz}, \mathrm{CDCl}_{3}\right) \delta$ 7.93-7.88 (m, $\left.2 \mathrm{H}\right)$, 7.55-7.50 (m, $1 \mathrm{H}), 7.45-7.39(\mathrm{~m}, 2 \mathrm{H}), 7.17-7.12(\mathrm{~m}, 2 \mathrm{H}), 6.85-6.80(\mathrm{~m}, 2 \mathrm{H}), 3.76(\mathrm{~s}, 3 \mathrm{H}), 3.28-3.14$ $(\mathrm{m}, 3 \mathrm{H}), 1.82-1.70(\mathrm{~m}, 1 \mathrm{H}), 1.66-1.54(\mathrm{~m}, 1 \mathrm{H}), 0.79(\mathrm{t}, J=7.2 \mathrm{~Hz}, 3 \mathrm{H}) ;{ }^{13} \mathrm{C}$ NMR $(100 \mathrm{MHz}$, $\left.\mathrm{CDCl}_{3}\right) \delta 199.5,158.0,137.3,136.7,132.9,128.6,128.5,128.1,113.8,55.2,45.9,42.3,29.4,12.2$; IR ( $\mathrm{NaCl}$ plates) 1685, 1510, $1245 \mathrm{~cm}^{-1}$; HRMS calcd for $\mathrm{C}_{18} \mathrm{H}_{21} \mathrm{O}_{2}[\mathrm{M}]^{+}$268.1463, found 268.1466; The enantiomeric excess was determined by HPLC (Chiralcel OJ, hexane/2-propanol $=199 / 1,1.0$ $\mathrm{mL} / \mathrm{min}, 254 \mathrm{~nm})$. Retention times: $28.9 \min$ [ $(S)$-enantiomer], $45.8 \mathrm{~min}$ [( $R)$-enantiomer].

3-(4-(Dimethylamino)phenyl)-1-phenylpentan-1-one. $[\alpha]_{\mathrm{D}}^{22}=-19.3^{\circ}\left(c=0.97, \mathrm{CHCl}_{3}\right) ;{ }^{1} \mathrm{H} \mathrm{NMR}$ $\left(400 \mathrm{MHz}, \mathrm{CDCl}_{3}\right) \delta$ 7.93-7.83 (m, 2H), 7.55-7.49 (m, 1H), 7.45-7.39 (m, 2H), 7.12-7.07 (m, 2H), 6.71-6.66 (m, 2H), 3.27-3.07 (m, 3H), $2.90(\mathrm{~s}, 6 \mathrm{H}), 1.80-1.69(\mathrm{~m}, 1 \mathrm{H}), 1.65-1.53(\mathrm{~m}, 1 \mathrm{H}), 0.79(\mathrm{t}, J$ $=7.4 \mathrm{~Hz}, 3 \mathrm{H}) ;{ }^{13} \mathrm{C}$ NMR $\left(100 \mathrm{MHz}, \mathrm{CDCl}_{3}\right) \delta 199.6,149.1,137.3,132.7,132.6,128.4,128.1,128.0$, 112.8, 46.0, 42.1, 40.7, 29.2, 12.1; IR (NaCl plates) 1680, 1615, 1520, 1450, $1350 \mathrm{~cm}^{-1}$; HRMS calcd for $\mathrm{C}_{19} \mathrm{H}_{23} \mathrm{NO}[\mathrm{M}]^{+} 281.1780$, found 281.1781; The enantiomeric excess was determined by HPLC (Chiralcel OJ, hexane/2-propanol $=95 / 5,0.5 \mathrm{~mL} / \mathrm{min}, 254 \mathrm{~nm}$ ). Retention time: $61.2 \mathrm{~min}$, 72.0 min.

3-(4-Chlorophenyl)-1-phenylpentan-1-one. ${ }^{1} \mathrm{H}$ NMR $\left(400 \mathrm{MHz}, \mathrm{CDCl}_{3}\right) \delta$ 7.92-7.87 (m, 2H), 7.57-7.52 (m, 1H), 7.46-7.40 (m, 2H), 7.27-7.22 (m, 2H), 7.18-7.13 (m, 2H), 3.31-3.18 (m, 3H), $1.83-1.70(\mathrm{~m}, 1 \mathrm{H}), 1.68-1.55(\mathrm{~m}, 1 \mathrm{H}), 0.80(\mathrm{t}, J=7.4 \mathrm{~Hz}, 3 \mathrm{H}) ;{ }^{13} \mathrm{C} \mathrm{NMR}\left(100 \mathrm{MHz}, \mathrm{CDCl}_{3}\right) \delta$ $198.8,143.1,137.1,133.0,131.8,129.0,128.6,128.5,128.0,45.4,42.3,29.2,12.0 ; \mathrm{IR}$ (NaCl plates) $1685,1490 \mathrm{~cm}^{-1}$; HRMS calcd for $\mathrm{C}_{17} \mathrm{H}_{18} \mathrm{ClO}[\mathrm{M}+\mathrm{H}]^{+}$273.1046, found 273.1042; The enantiomeric excess was determined by HPLC (Chiralcel OJ, hexane/2-propanol $=199 / 1,0.3 \mathrm{~mL} / \mathrm{min}, 254 \mathrm{~nm}$ ). Retention time: $53.6 \mathrm{~min}[(S)$-enantiomer], $57.5 \min [(R)$-enantiomer].

3-(4-(Trifluoromethyl)phenyl)-1-phenylpentan-1-one. $[\alpha]_{\mathrm{D}}{ }^{22}=+5.9^{\circ}\left(c=1.39, \mathrm{CHCl}_{3}\right) ;{ }^{1} \mathrm{H} \mathrm{NMR}$ (400 MHz, $\left.\mathrm{CDCl}_{3}\right) \delta$ 7.92-7.87 (m, 2H), 7.57-7.50 (m, 3H), 7.46-7.40 (m, 2H), 7.37-7.32 (d, J=8 Hz, $2 \mathrm{H}), 3.38-3.24(\mathrm{~m}, 3 \mathrm{H}), 1.88-1.76(\mathrm{~m}, 1 \mathrm{H}), 1.72-1.59(\mathrm{~m}, 1 \mathrm{H}), 0.81(\mathrm{t}, J=7.4 \mathrm{~Hz}, 3 \mathrm{H}) ;{ }^{13} \mathrm{C}$ NMR $\left(100 \mathrm{MHz}, \mathrm{CDCl}_{3}\right) \delta 198.5,148.8,137.0,133.1,128.6,128.0,127.9,125.3$ (q, $\left.J=38.3 \mathrm{~Hz}\right), 45.1$, 42.6, 29.1, 11.9; IR (NaCl plates) 1685, 1325, 1165, 1120, $1070 \mathrm{~cm}^{-1}$; HRMS calcd for $\mathrm{C}_{18} \mathrm{H}_{18} \mathrm{~F}_{3} \mathrm{O}$ $[\mathrm{M}+\mathrm{H}]^{+}$307.1310, found 307.1303; The enantiomeric excess was determined by HPLC (Chiralcel OJ, hexane, $0.5 \mathrm{~mL} / \mathrm{min}, 254 \mathrm{~nm}$ ). Retention time: $58.2 \mathrm{~min}, 74.0 \mathrm{~min}$.

3-(1-Naphthyl)-1-phenylpentan-1-one. $[\alpha]_{\mathrm{D}}{ }^{22}=+45.6^{\circ}\left(c=1.16, \mathrm{CHCl}_{3}\right) ;{ }^{1} \mathrm{H}$ NMR $(400 \mathrm{MHz}$, $\left.\mathrm{CDCl}_{3}\right) \delta 8.24(\mathrm{~d}, J=8.3 \mathrm{~Hz}, 1 \mathrm{H}), 7.94-7.88(\mathrm{~m}, 2 \mathrm{H}), 7.87-7.82(\mathrm{~m}, 1 \mathrm{H}), 7.71(\mathrm{dd}, J=1.7,7.3,1 \mathrm{H})$, 7.55-7.37 (m, 7H), 4.32-4.20 (m, 1H), 3.45-3.31 (m, 2H), 2.00-1.80 (m, 2H), $0.84(\mathrm{t}, J=7.4 \mathrm{~Hz}$, $3 \mathrm{H}) ;{ }^{13} \mathrm{C}$ NMR $\left(100 \mathrm{MHz}, \mathrm{CDCl}_{3}\right) \delta$ 199.2, 141.0, 137.2, 134.0, 132.9, 132.1, 128.9, 128.5, 128.0, 126.7, 125.9, 125.4, 123.4, 45.4, 28.6, 12.0; IR (NaCl plates) 1680, 775, 755, $690 \mathrm{~cm}^{-1}$; HRMS calcd for $\mathrm{C}_{21} \mathrm{H}_{20} \mathrm{O}[\mathrm{M}]^{+}$288.1514, found 288.1529; The enantiomeric excess was determined by HPLC $($ Chiralcel OJ, hexane $/ 2$-propanol $=199 / 1,0.5 \mathrm{~mL} / \mathrm{min}, 254 \mathrm{~nm})$. Retention time: $45.0 \mathrm{~min}, 60.2$ $\min$.

3-Ethyl-1-phenyloctan-1-one. $[\alpha]_{\mathrm{D}}^{22}=-1.8^{\circ}\left(c=1.15, \mathrm{CHCl}_{3}\right) ;{ }^{1} \mathrm{H}$ NMR $\left(400 \mathrm{MHz}, \mathrm{CDCl}_{3}\right) \delta$ 7.98-7.93 (m, 2H), 7.57-7.51 (m, 1H), 7.47-7.42 (m, 2H), 2.93-2.81 (m, 2H), 2.09-2.00 (m, $1 \mathrm{H})$, 1.46-1.19 (m, 10H), 0.91-0.84 (m, 6H); ${ }^{13} \mathrm{C}$ NMR (100 MHz, $\left.\mathrm{CDCl}_{3}\right) \delta 200.7,137.5,132.7,128.5$, 128.0, 43.0, 35.7, 33.5, 32.1, 26.5, 26.3, 22.6, 14.0, 10.9; IR (NaCl plates) 2925, 2860, 1685, 1450, $750,690 \mathrm{~cm}^{-1}$; HRMS calcd for $\mathrm{C}_{16} \mathrm{H}_{25} \mathrm{O}[\mathrm{M}+\mathrm{H}]^{+}$233.1905, found 233.1909; The enantiomeric 
excess was determined by HPLC (Chiralcel OD-H, hexane, $0.5 \mathrm{~mL} / \mathrm{min}, 254 \mathrm{~nm}$ ). Retention time: $40.7 \mathrm{~min}, 44.0 \mathrm{~min}$.

3-Ethyl-4-methyl-1-phenylpentan-1-one. $[\alpha]_{\mathrm{D}}^{22}=+4.3^{\circ}\left(c=1.06, \mathrm{CHCl}_{3}\right) ;{ }^{1} \mathrm{H}$ NMR $(400 \mathrm{MHz}$, $\left.\mathrm{CDCl}_{3}\right) \delta$ 7.99-7.93 (m, 2H), 7.57-7.51 (m, 1H), 7.49-7.42 (m, 2H), 2.91 (dd, J=16.1, 5.6 Hz, H), $2.78(\mathrm{dd}, J=16.1,7.5 \mathrm{~Hz}, 1 \mathrm{H}), 2.02-1.94(\mathrm{~m}, 1 \mathrm{H}), 1.85-1.74(\mathrm{~m}, 1 \mathrm{H}), 1.46-1.21(\mathrm{~m}, 2 \mathrm{H}), 0.93-0.85$ (m, 9H); ${ }^{13} \mathrm{C}$ NMR $\left(100 \mathrm{MHz}, \mathrm{CDCl}_{3}\right) \delta 201.0,137.5,132.8,128.5,128.1,41.5,40.0,29.4,23.9$, 19.6, 18.6, 11.9; IR ( $\mathrm{NaCl}$ plates) 2960, $1680 \mathrm{~cm}^{-1}$; HRMS calcd for $\mathrm{C}_{14} \mathrm{H}_{21} \mathrm{O}[\mathrm{M}+\mathrm{H}]^{+} 205.1592$, found 205.1587; The enantiomeric excess was determined by GLC (Chirasil-DEX CB (30 m), 120 ${ }^{\circ} \mathrm{C}, 35 \mathrm{~cm} / \mathrm{sec}$ ). Retention time: $47.8 \mathrm{~min}, 48.8 \mathrm{~min}$.

3-Ethyl-4,4-dimethyl-1-phenylpentan-1-one. $[\alpha]_{\mathrm{D}}^{22}=-5.3^{\circ}\left(c=0.83, \mathrm{CHCl}_{3}\right) ;{ }^{1} \mathrm{H}$ NMR $(400$ $\left.\mathrm{MHz}, \mathrm{CDCl}_{3}\right) \delta$ 8.01-7.88 (m, 2H), 7.58-7.52 (m, $\left.1 \mathrm{H}\right), 7.50-7.43(\mathrm{~m}, 2 \mathrm{H}), 3.06(\mathrm{dd}, J=17.3,4.6 \mathrm{~Hz}$, $1 \mathrm{H}), 2.71(\mathrm{dd}, J=17.3,6.5 \mathrm{~Hz}, 1 \mathrm{H}), 2.05-1.95(\mathrm{~m}, 1 \mathrm{H}), 1.71-1.59(\mathrm{~m}, 1 \mathrm{H}), 1.15-1.02(\mathrm{~m}, 1 \mathrm{H}), 0.90$ (s, 9H), $0.83(\mathrm{t}, J=7.5 \mathrm{~Hz}, 3 \mathrm{H}) ;{ }^{13} \mathrm{C}$ NMR $\left(100 \mathrm{MHz}, \mathrm{CDCl}_{3}\right) \delta$ 200.7, 137.5, 132.7, 128.5, 128.0, 44.9, 40.0, 33.6, 27.6, 23.9, 13.5; IR ( $\mathrm{NaCl}$ plates) 2960, $1690 \mathrm{~cm}^{-1}$; HRMS calcd for $\mathrm{C}_{15} \mathrm{H}_{23} \mathrm{O}$ $[\mathrm{M}+\mathrm{H}]^{+}$219.1749, found 219.1756; The enantiomeric excess was determined by GLC (ChirasilDEX CB $\left.(30 \mathrm{~m}), 120^{\circ} \mathrm{C}, 35 \mathrm{~cm} / \mathrm{sec}\right)$. Retention time: $65.3 \mathrm{~min}, 66.8 \mathrm{~min}$.

1-(4-Methoxyphenyl)-3-phenylpentan-1-one. $[\alpha]_{\mathrm{D}}{ }^{22}=+7.9^{\circ}\left(c=1.01, \mathrm{CHCl}_{3}\right) ;{ }^{1} \mathrm{H}$ NMR $(400$ $\left.\mathrm{MHz}, \mathrm{CDCl}_{3}\right) \delta$ 7.93-7.85 (m, 2H), 7.31-7.14 (m, 5H), 6.93-9.87 (m, 2H), $3.85(\mathrm{~s}, 3 \mathrm{H}), 3.27-3.14(\mathrm{~m}$, $3 \mathrm{H}), 1.84-1.72(\mathrm{~m}, 1 \mathrm{H}), 1.69-1.55(\mathrm{~m}, 1 \mathrm{H}), 0.79(\mathrm{t}, J=7.4 \mathrm{~Hz}, 3 \mathrm{H}) ;{ }^{13} \mathrm{C}$ NMR $\left(100 \mathrm{MHz}, \mathrm{CDCl}_{3}\right) \delta$ $197.8,163.3,144.8,130.3,128.3,127.6,126.2,113.6,55.4,45.3,43.2,29.2,12.1$; IR (KBr) 1670, $1605,1260,1175,700 \mathrm{~cm}^{-1} ; \mathrm{mp}=61-63{ }^{\circ} \mathrm{C}$; HRMS calcd for $\mathrm{C}_{18} \mathrm{H}_{21} \mathrm{O}_{2}[\mathrm{M}+\mathrm{H}]^{+}$269.1542, found 269.1548; The enantiomeric excess was determined by HPLC (Chiralcel OJ, hexane/2-propanol = 9/1, $0.5 \mathrm{~mL} / \mathrm{min}, 254 \mathrm{~nm}$ ). Retention time: $44.9 \mathrm{~min}, 51.1 \mathrm{~min}$.

1-(4-Chlorophenyl)-3-phenylpentan-1-one. $[\alpha]_{\mathrm{D}}{ }^{22}=+3.1^{\circ}\left(c=1.21, \mathrm{CHCl}_{3}\right) ;{ }^{1} \mathrm{H}$ NMR $(400 \mathrm{MHz}$, $\left.\mathrm{CDCl}_{3}\right) \delta$ 7.85-7.78 (m, 2H), 7.41-7.35 (m, 2H), 7.31-7.14 (m, 5H), 3.30-3.14 (m, 3H), 1.83-1.72 (m, $1 \mathrm{H}), 1.70-1.58(\mathrm{~m}, 1 \mathrm{H}), 0.80(\mathrm{t}, J=7.3 \mathrm{~Hz}, 3 \mathrm{H}) ;{ }^{13} \mathrm{C} \mathrm{NMR}\left(100 \mathrm{MHz}, \mathrm{CDCl}_{3}\right) \delta 198.0,144.3,139.2$, 135.5, 129.4, 128.8, 128.4, 127.5, 126.3, 45.5, 43.0, 29.2, 12.0; IR (NaCl plates) 1690, 1590, 1090, 1015, 760, $700 \mathrm{~cm}^{-1}$; HRMS calcd for $\mathrm{C}_{17} \mathrm{H}_{18} \mathrm{ClO}[\mathrm{M}+\mathrm{H}]^{+}$273.1046, found 273.1032; The enantiomeric excess was determined by HPLC (Chiralcel OD-H, hexane/2-propanol $=98 / 2,0.5$ $\mathrm{mL} / \mathrm{min}, 254 \mathrm{~nm}$ ). Retention time: $16.7 \mathrm{~min}, 18.5 \mathrm{~min}$.

2-Methyl-6-phenyloctan-4-one. $[\alpha]_{\mathrm{D}}^{22}=-34.2^{\circ}\left(c=1.20, \mathrm{CHCl}_{3}\right) ;{ }^{1} \mathrm{H}$ NMR $\left(400 \mathrm{MHz}, \mathrm{CDCl}_{3}\right) \delta$ 7.30-7.23 (m, 2H), 7.20-7.12 (m, 3H), 3.10-3.00 (m, 1H), 2.73-2.60 (m, 2H), 2.21-1.97 (m, 3H), 1.72-1.49 (m, 2H), 0.82-0.74 (m, 9H); ${ }^{13} \mathrm{C}$ NMR (100 MHz, $\left.\mathrm{CDCl}_{3}\right) \delta 209.7,144.3,128.3,127.5$, 126.1, 52.5, 50.0, 42.7, 29.2, 24.2, 22.4, 22.3, 11.9; IR (NaCl plates) 2960, 1715, $700 \mathrm{~cm}^{-1}$; HRMS calcd for $\mathrm{C}_{15} \mathrm{H}_{23} \mathrm{O}[\mathrm{M}+\mathrm{H}]^{+}$219.1749, found 219.1737; The enantiomeric excess was determined by HPLC (Chiralcel OJ, hexane/2-propanol $=98 / 2,0.5 \mathrm{~mL} / \mathrm{min}, 254 \mathrm{~nm}$ ). Retention time: $14.3 \mathrm{~min}$, $16.1 \mathrm{~min}$.

1-Cyclohexyl-3-phenylpentan-1-one. $[\alpha]_{\mathrm{D}}^{22}=-24.0^{\circ}\left(c=1.20, \mathrm{CHCl}_{3}\right) ;{ }^{1} \mathrm{H}$ NMR $(400 \mathrm{MHz}$, $\mathrm{CDCl}_{3}$ ) $\delta$ 7.30-7.22 (m, 2H), 7.20-7.13 (m, 3H), 3.11-3.00 (m, 1H), 2.78-2.66 (m, 2H), 2.14-2.23 (m, $1 \mathrm{H}), 1.80-1.48(\mathrm{~m}, 7 \mathrm{H}), 1.30-1.09(\mathrm{~m}, 5 \mathrm{H}), 0.76(\mathrm{t}, J=7.4 \mathrm{~Hz}, 3 \mathrm{H}) ;{ }^{13} \mathrm{C} \mathrm{NMR}\left(100 \mathrm{MHz}, \mathrm{CDCl}_{3}\right) \delta$ $212.9,144.6,128.2,127.5,126.1,51.2,47.7,42.5,29.1,28.1,28.0,25.7,25.6,25.5,12.0 ; \mathrm{IR}(\mathrm{NaCl}$ plates) 2930, $1710 \mathrm{~cm}^{-1}$; HRMS calcd for $\mathrm{C}_{17} \mathrm{H}_{25} \mathrm{O}[\mathrm{M}+\mathrm{H}]^{+}$245.1905, found 245.1892; The enantiomeric excess was determined by HPLC (Chiralcel OJ, hexane/2-propanol $=98 / 2,0.5$ $\mathrm{mL} / \mathrm{min}, 254 \mathrm{~nm}$ ). Retention time: $15.3 \mathrm{~min}, 16.8$ min.

3-Phenylundecan-5-one. $[\alpha]_{\mathrm{D}}^{22}=-32.8^{\circ}\left(c=1.69, \mathrm{CHCl}_{3}\right)$; ${ }^{1} \mathrm{H}$ NMR $\left(400 \mathrm{MHz}, \mathrm{CDCl}_{3}\right) \delta 7.30$ $7.24(\mathrm{~m}, 2 \mathrm{H}), 7.19-7.13(\mathrm{~m}, 3 \mathrm{H}), 3.08-3.00(\mathrm{~m}, 1 \mathrm{H}), 2.75-2.62(\mathrm{~m}, 2 \mathrm{H}), 2.34-2.20(\mathrm{~m}, 2 \mathrm{H}), 1.72-1.50$ 
(m, 2H), 1.44 (quint, $J=7.4,2 \mathrm{H}), 1.30-1.10(\mathrm{~m}, 6 \mathrm{H}), 0.83(\mathrm{t}, J=7.1,3 \mathrm{H}), 0.77(\mathrm{t}, J=7.5,3 \mathrm{H}) ;{ }^{13} \mathrm{C}$ NMR (100 MHz, $\left.\mathrm{CDCl}_{3}\right) \delta 210.3,144.4,128.3,127.5,126.2,49.6,43.5,42.9,31.5,29.2,28.7,23.4$, 14.0, 11.9; IR ( $\mathrm{NaCl}$ plates) 2930, $1715 \mathrm{~cm}^{-1}$; HRMS calcd for $\mathrm{C}_{17} \mathrm{H}_{23} \mathrm{O}[\mathrm{M}+\mathrm{H}]^{+} 247.2062$, found 247.2074; The enantiomeric excess was determined by HPLC (Chiralcel OJ, hexane/2-propanol = 98/2, $0.5 \mathrm{~mL} / \mathrm{min}, 254 \mathrm{~nm})$. Retention time: $13.7 \mathrm{~min}, 15.9 \mathrm{~min}$.

3-Methylundecan-5-one. $[\alpha]_{\mathrm{D}}{ }^{22}=-3.0^{\circ}\left(c=1.53, \mathrm{CHCl}_{3}\right) ;{ }^{1} \mathrm{H} \mathrm{NMR}\left(400 \mathrm{MHz}, \mathrm{CDCl}_{3}\right) \delta$ 2.42-2.34 $(\mathrm{m}, 3 \mathrm{H}), 2.19(\mathrm{dd}, J=8.0 \mathrm{~Hz}, 15.9,1 \mathrm{H}), 1.98-1.86(\mathrm{~m}, 1 \mathrm{H}), 1.60-1.50(\mathrm{~m}, 2 \mathrm{H}), 1.37-1.13(\mathrm{~m}, 8 \mathrm{H})$, 0.93-0.82 (m, 9H); ${ }^{13} \mathrm{C}$ NMR (100 MHz, $\left.\mathrm{CDCl}_{3}\right) \delta$ 211.5, 49.9, 43.4, 31.6, 30.8, 29.5, 28.9, 23.7, 22.5, 19.3, 14.0, 11.3; IR ( $\mathrm{NaCl}$ plates) 2960, 2930, $1715 \mathrm{~cm}^{-1}$; HRMS calcd for $\mathrm{C}_{12} \mathrm{H}_{24} \mathrm{O}[\mathrm{M}]^{+}$ 184.1827, found 184.1810; The enantiomeric excess was determined by GLC (Chiraldex G-TA (30 $\mathrm{m}), 40^{\circ} \mathrm{C}, 80 \mathrm{~cm} / \mathrm{sec}$ ). Retention time: $726 \mathrm{~min}, 773 \mathrm{~min}$.

4-Methylnonan-2-one. ${ }^{1} \mathrm{H}$ NMR $\left(400 \mathrm{MHz}, \mathrm{CDCl}_{3}\right) \delta 2.83$ (d, $\left.J=6.8 \mathrm{~Hz}, 2 \mathrm{H}\right), 2.14(\mathrm{~s}, 3 \mathrm{H}), 1.90-$ $1.81(\mathrm{~m}, 1 \mathrm{H}), 1.38-1.15(\mathrm{~m}, 10 \mathrm{H}), 0.88(\mathrm{t}, J=6.8 \mathrm{~Hz}, 3 \mathrm{H}), 0.85(\mathrm{t}, J=7.6 \mathrm{~Hz}, 3 \mathrm{H}) ;{ }^{13} \mathrm{C}$ NMR $(100$ $\left.\mathrm{MHz}, \mathrm{CDCl}_{3}\right) \delta 209.4,48.3,35.2,33.4,32.1,30.4,26.3,26.2,22.6,14.0,10.7$; IR (NaCl plates) 2960, 2930, $1720 \mathrm{~cm}^{-1}$; HRMS calcd for $\mathrm{C}_{11} \mathrm{H}_{22} \mathrm{O}[\mathrm{M}]^{+}$170.1671, found 170.1683; The enantiomeric excess was determined by GLC (Chiraldex G-TA $(30 \mathrm{~m}), 70{ }^{\circ} \mathrm{C}, 40 \mathrm{~cm} / \mathrm{sec}$ ). Retention time: 81.6 $\min [(R)$-enantiomer], $83.6 \min [(S)$-enantiomer $]$.

(Z)-1,3-Diphenylpropenone. ${ }^{1} \mathrm{H}$ NMR $\left(400 \mathrm{MHz}, \mathrm{CDCl}_{3}\right) \delta$ 7.98-7.94 (m, 2H), 7.55-7.48 (m, $\left.1 \mathrm{H}\right)$, 7.44-7.37 (m, 4H), 7.26-7.19 (m, 3H), $7.02(\mathrm{~d}, J=12.9 \mathrm{~Hz}, 1 \mathrm{H}), 6.62(\mathrm{~d}, J=12.9 \mathrm{~Hz}, 1 \mathrm{H}) ;{ }^{13} \mathrm{C} \mathrm{NMR}$ $\left(100 \mathrm{MHz}, \mathrm{CDCl}_{3}\right) \delta 194.7,139.4,137.1,135.1,133.2,129.4,129.0,128.8,128.5,128.2,126.7$; IR (KBr) $1665,1595,1225,690 \mathrm{~cm}^{-1} ; \mathrm{mp}=43-45{ }^{\circ} \mathrm{C}$; HRMS calcd for $\mathrm{C}_{15} \mathrm{H}_{13} \mathrm{O}[\mathrm{M}+\mathrm{H}]^{+} 209.0966$, found 209.0966. 


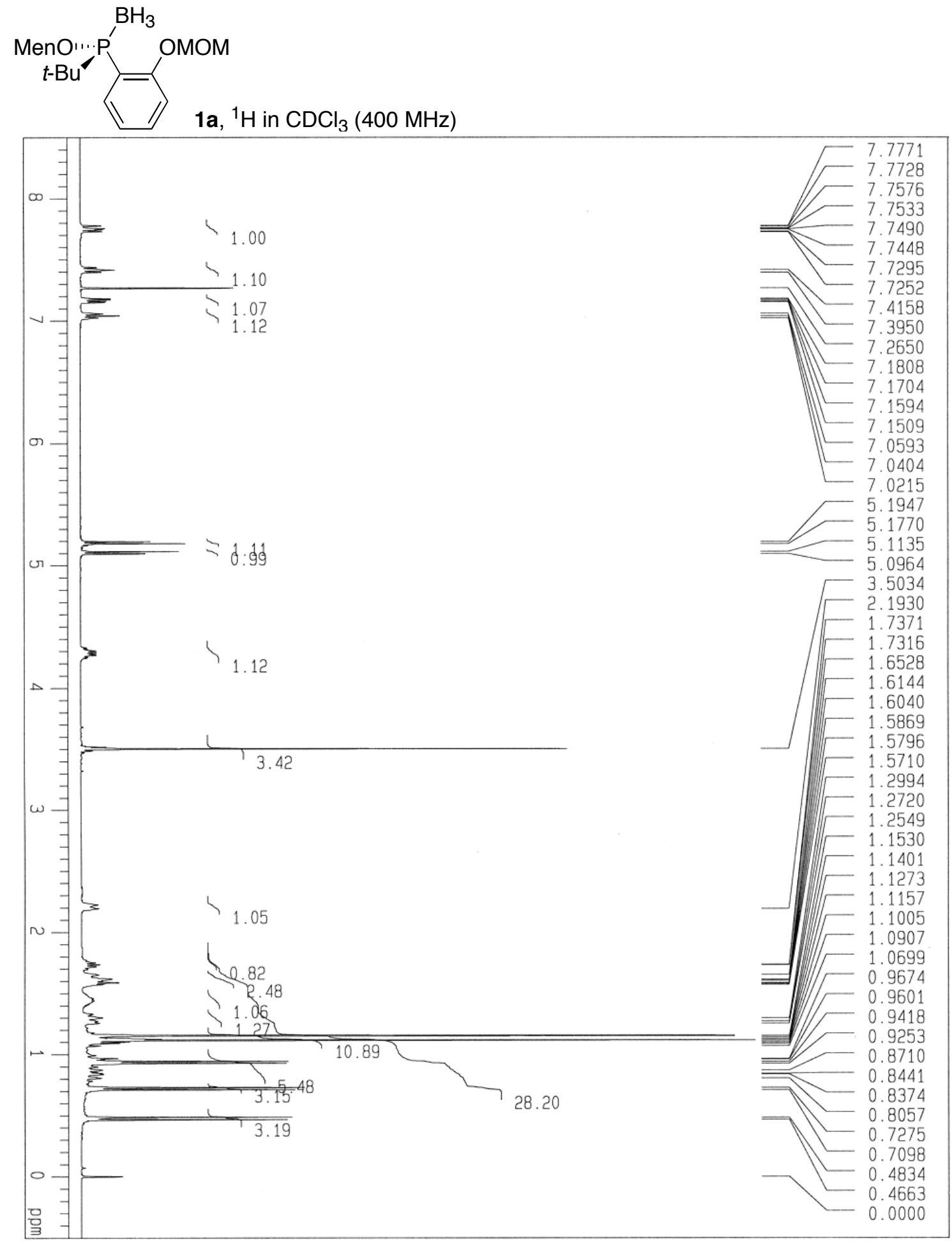




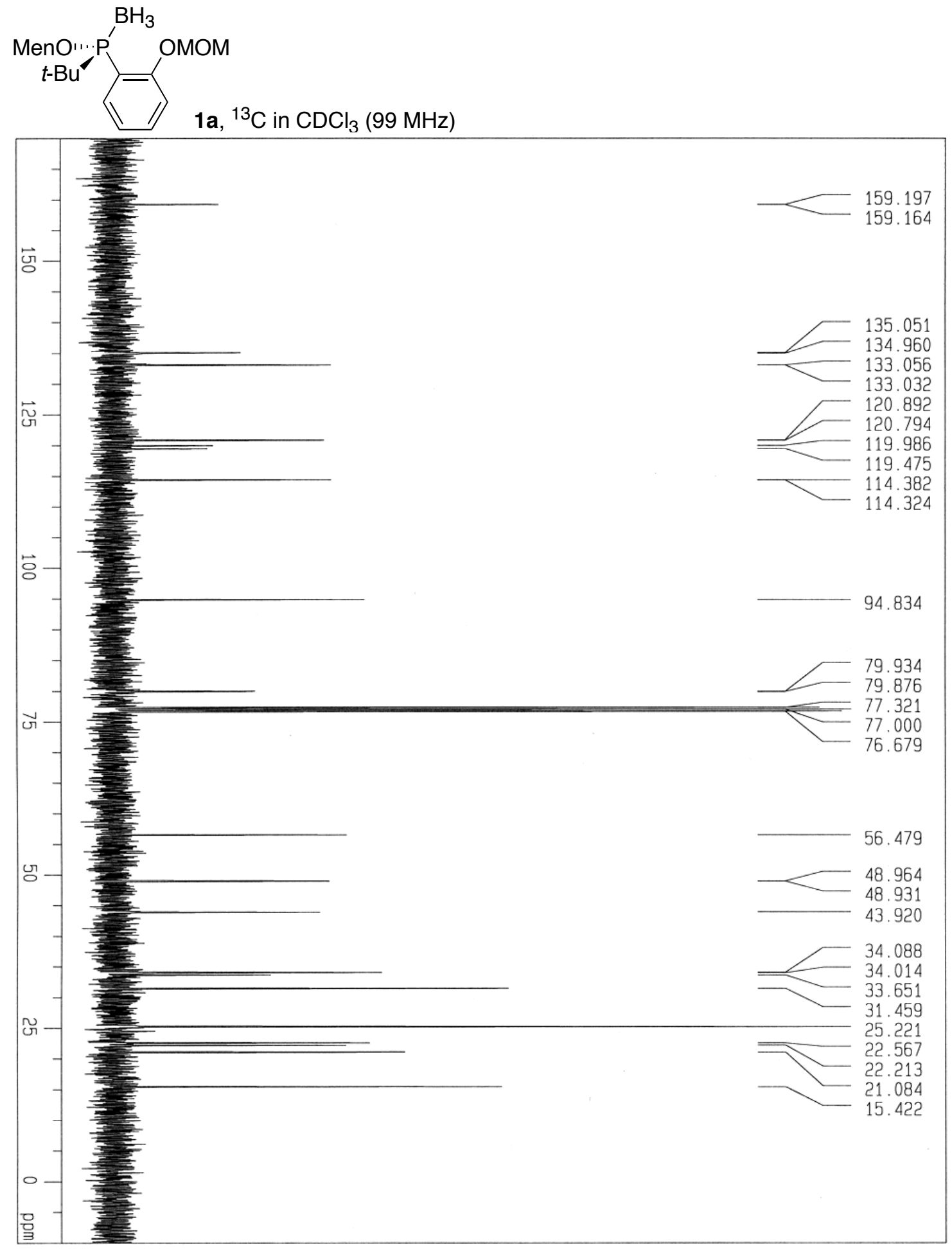




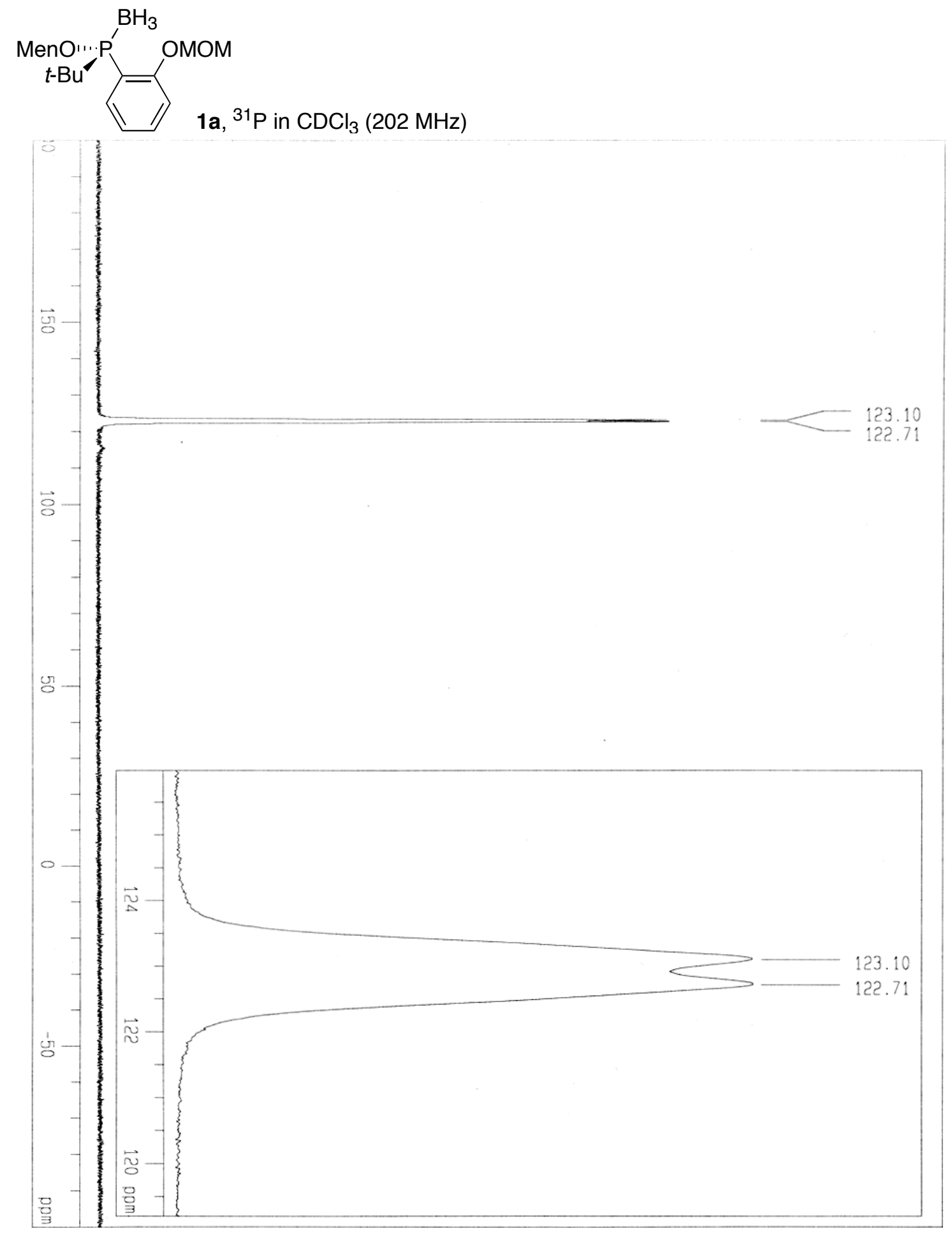




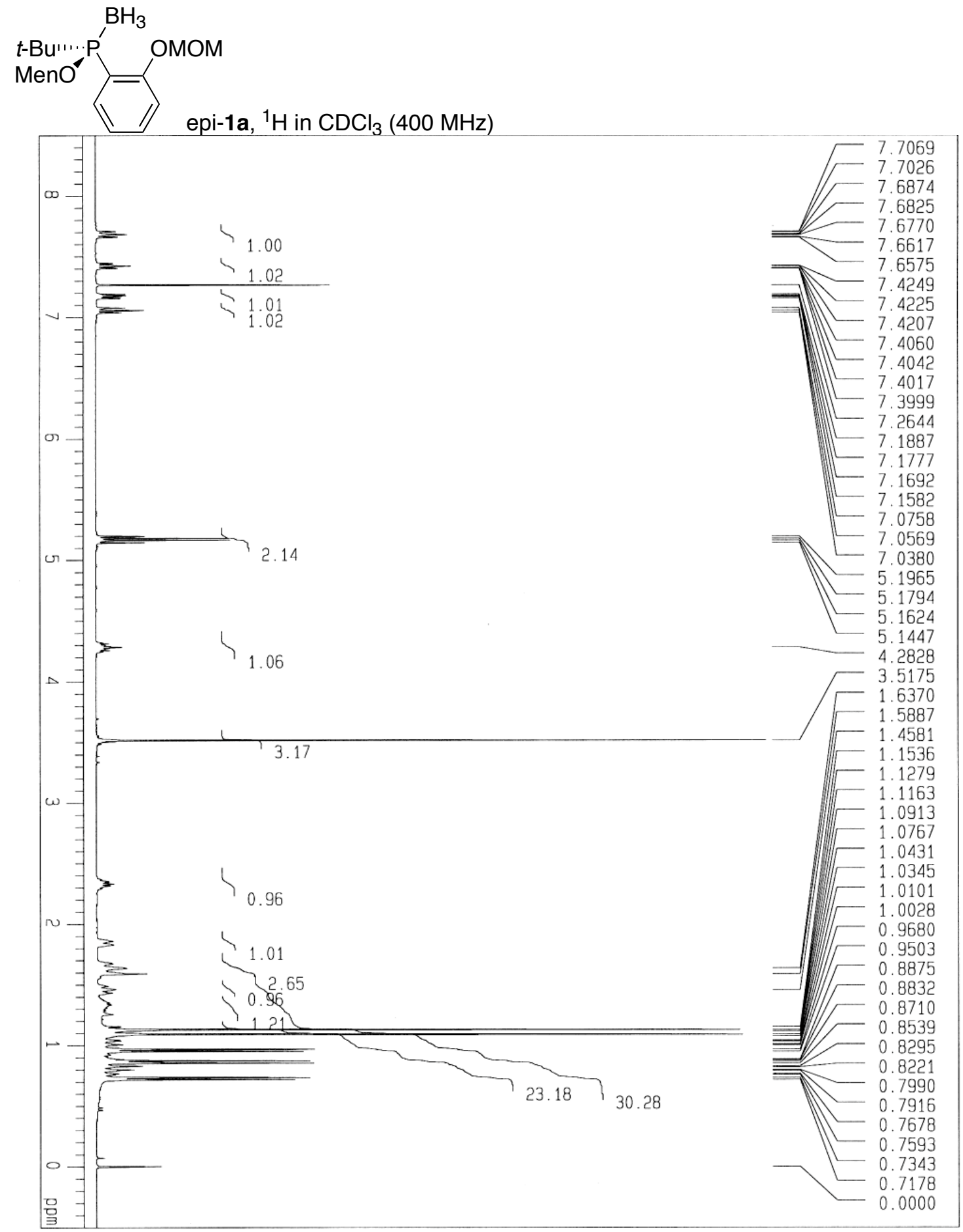




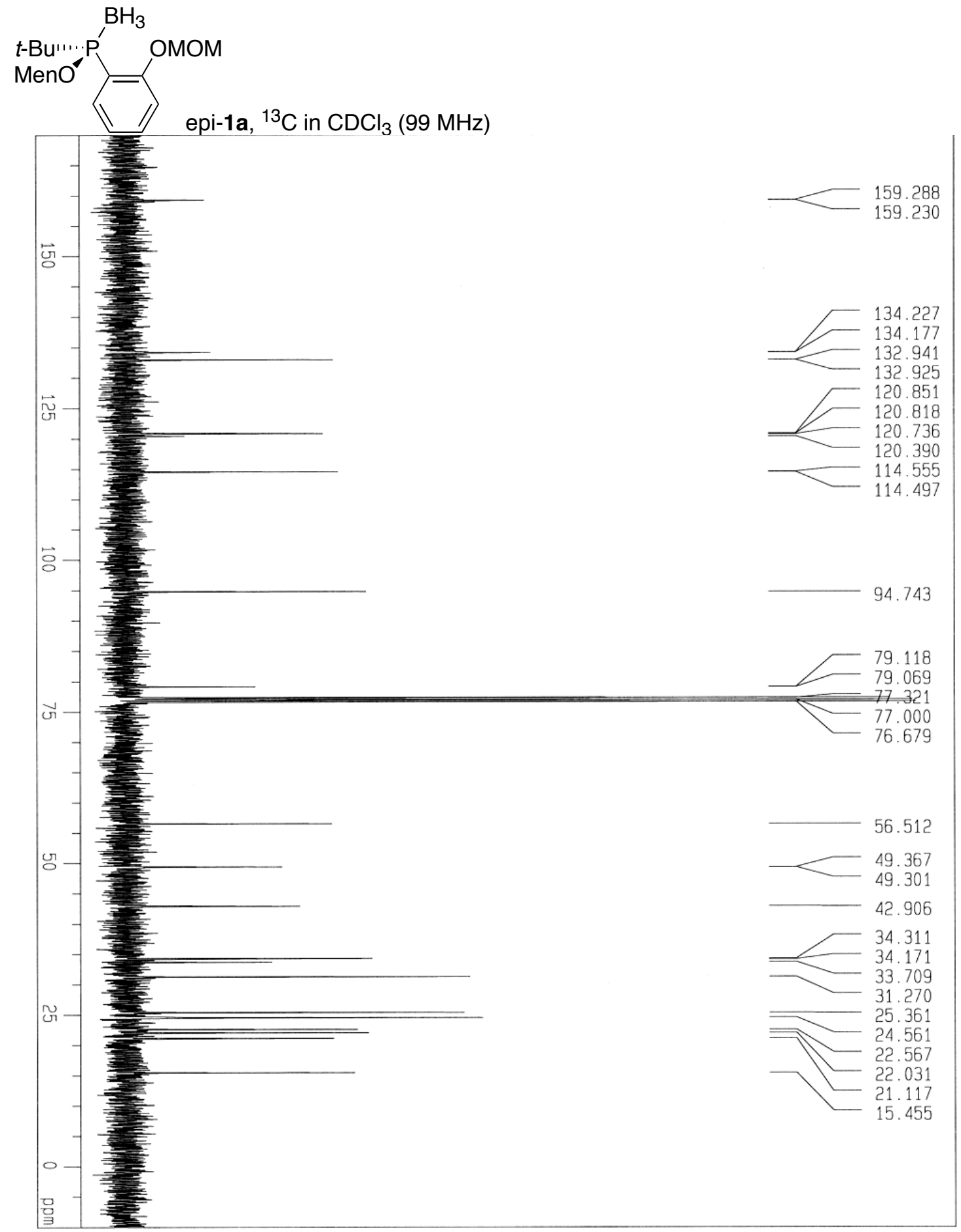




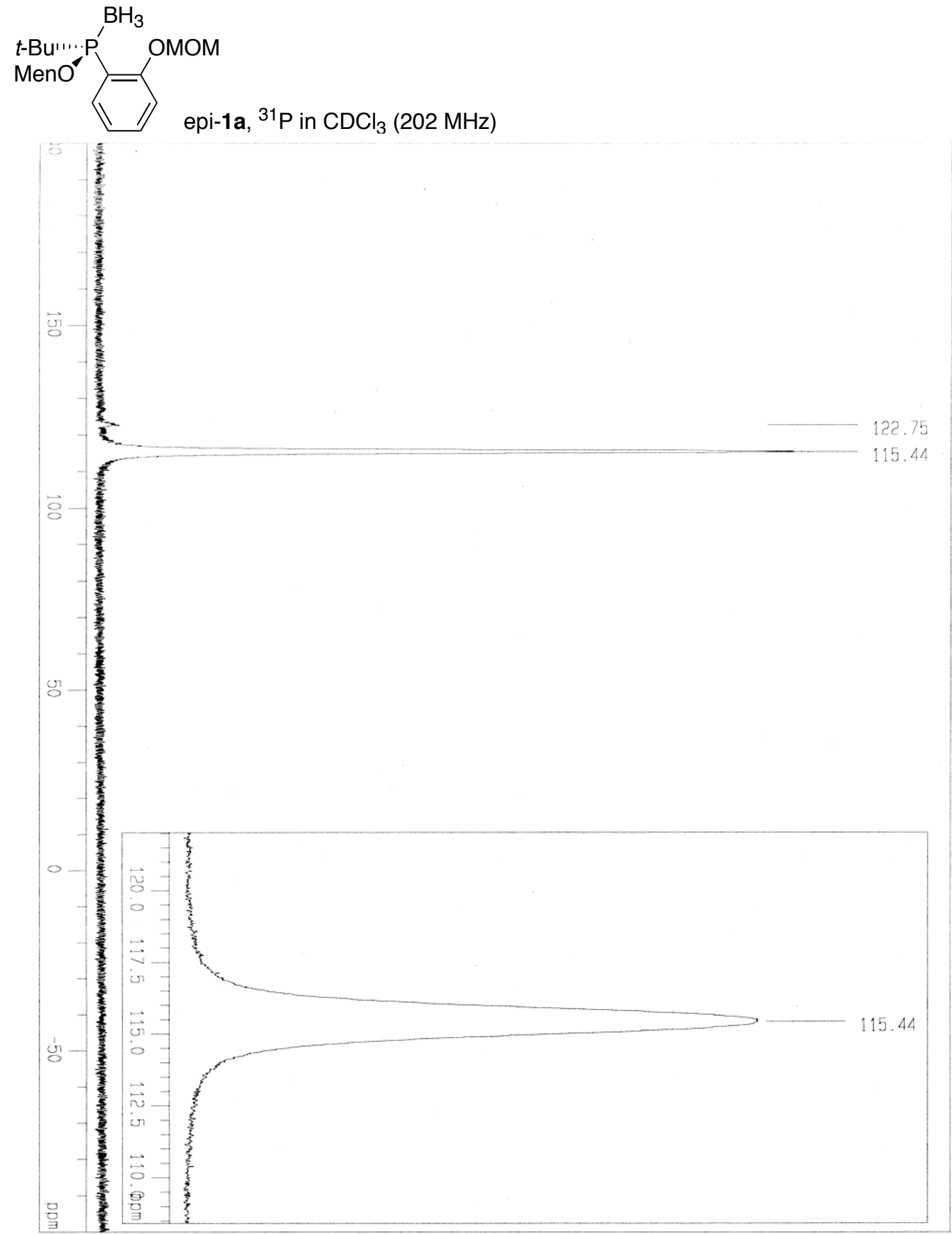




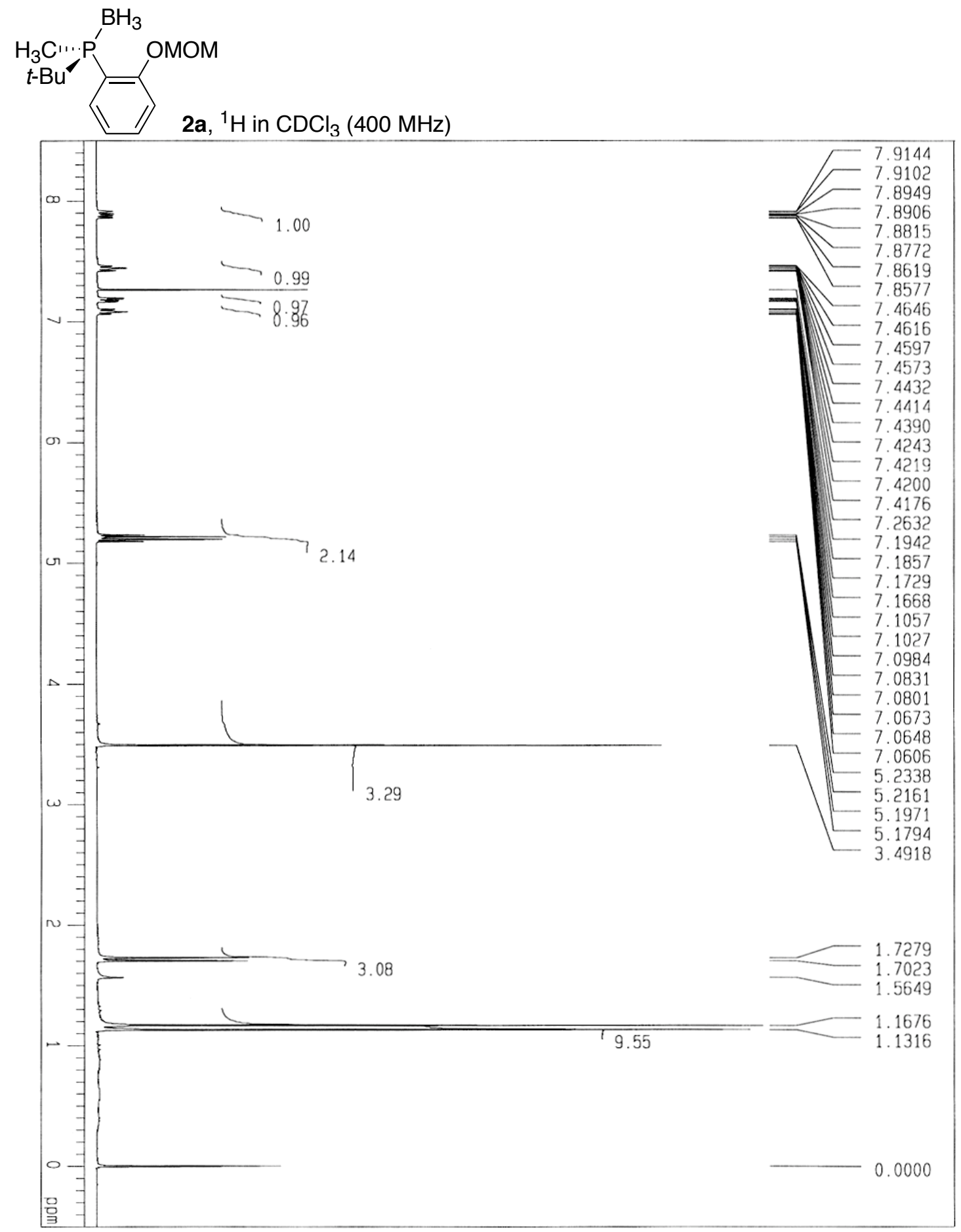




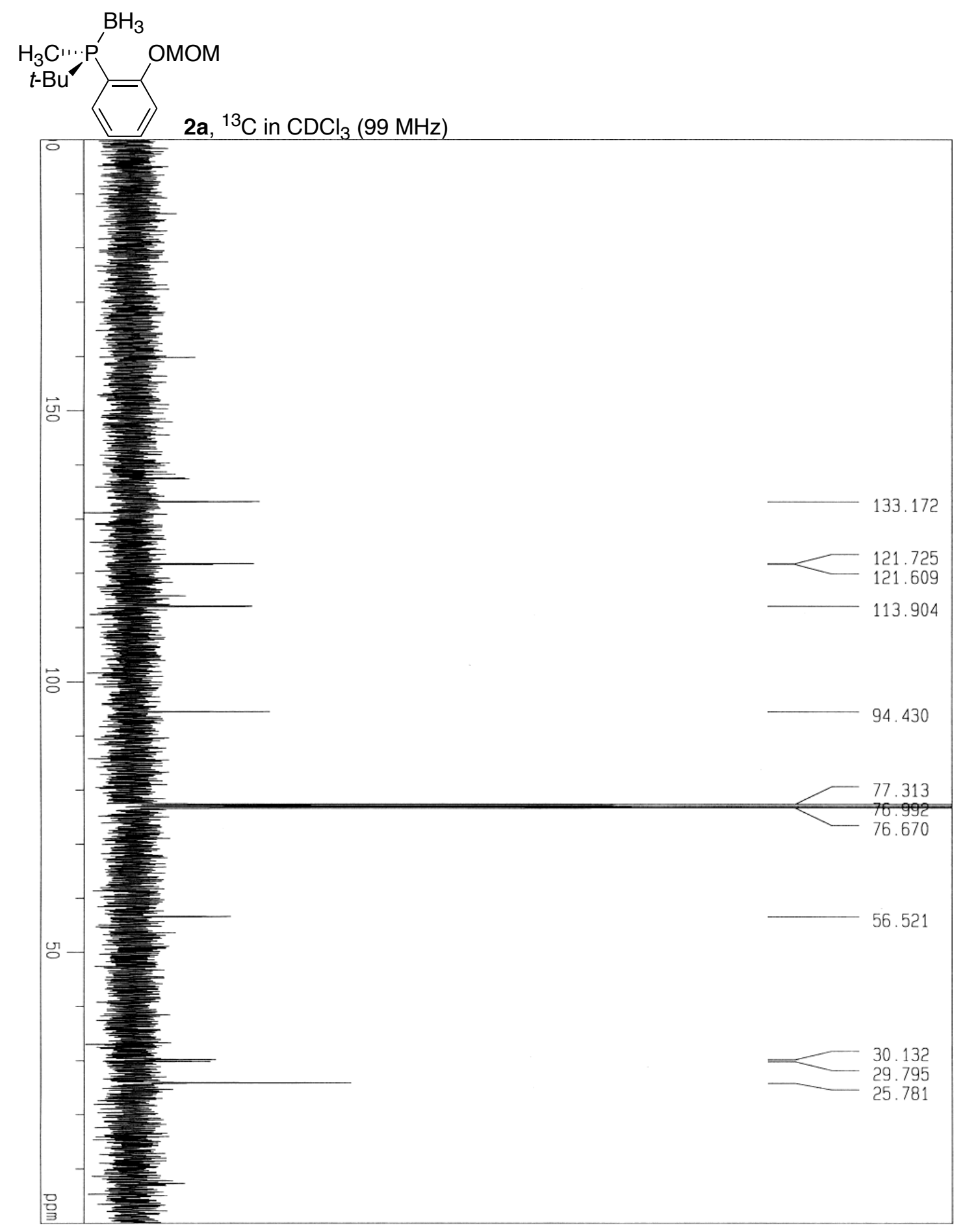




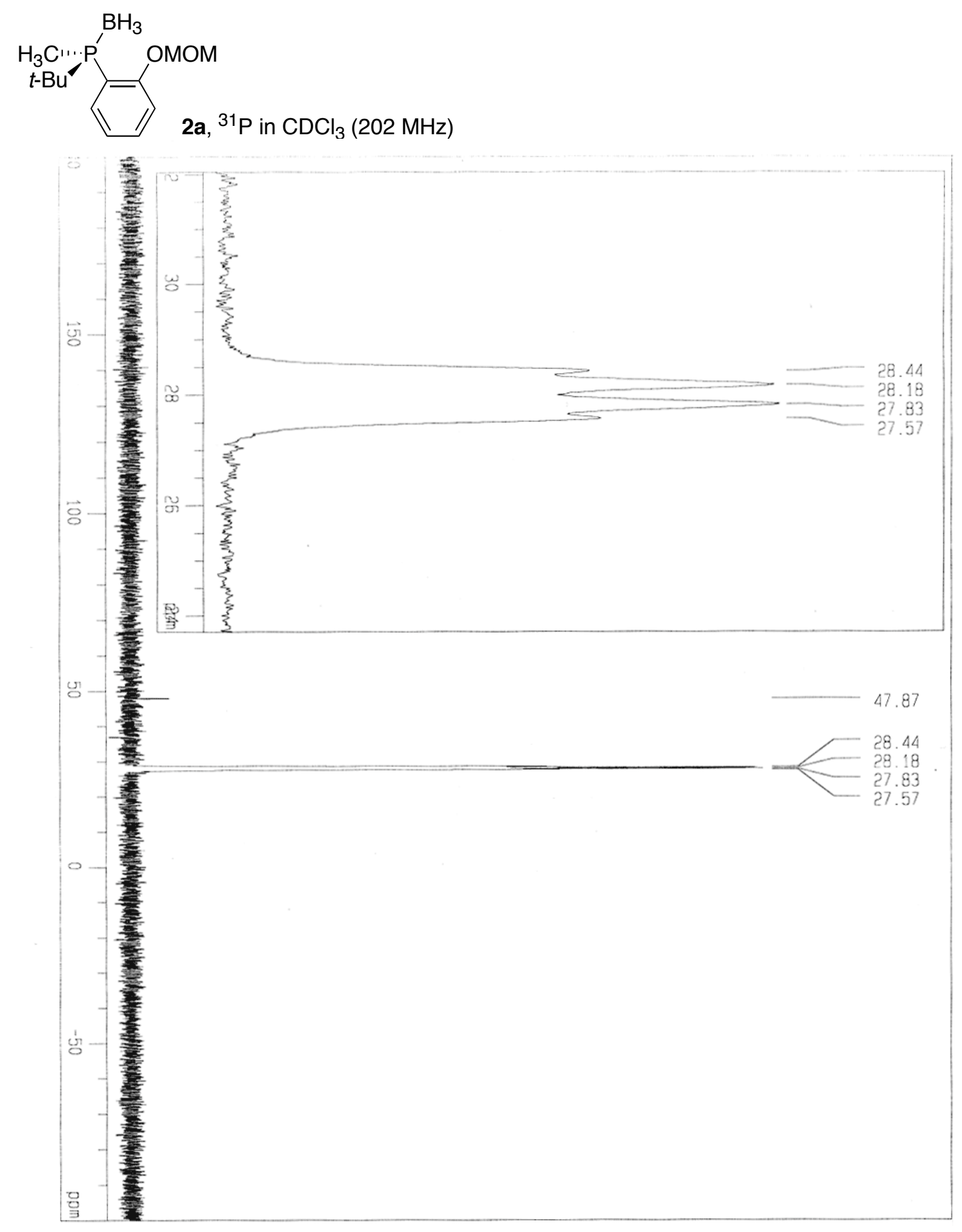




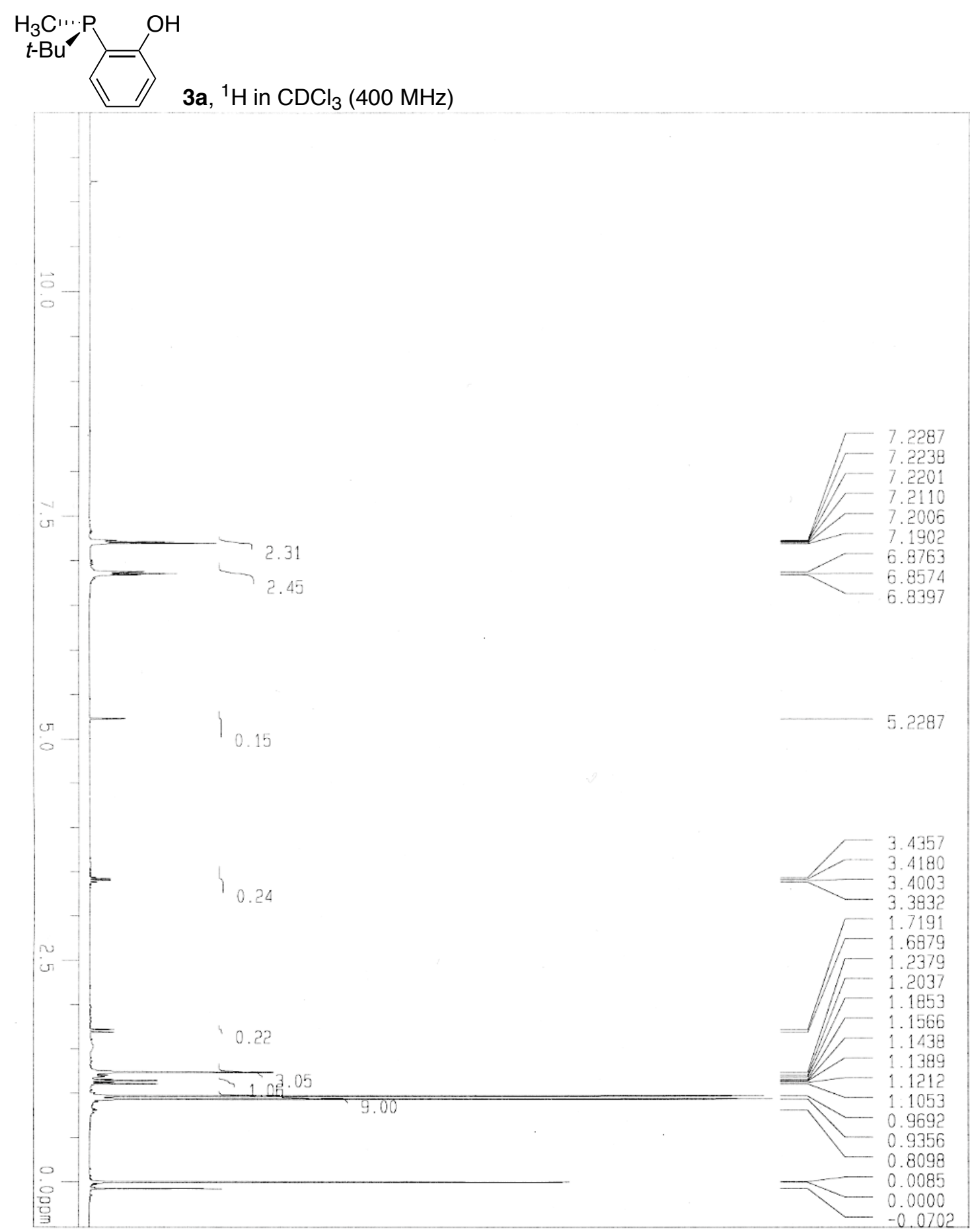




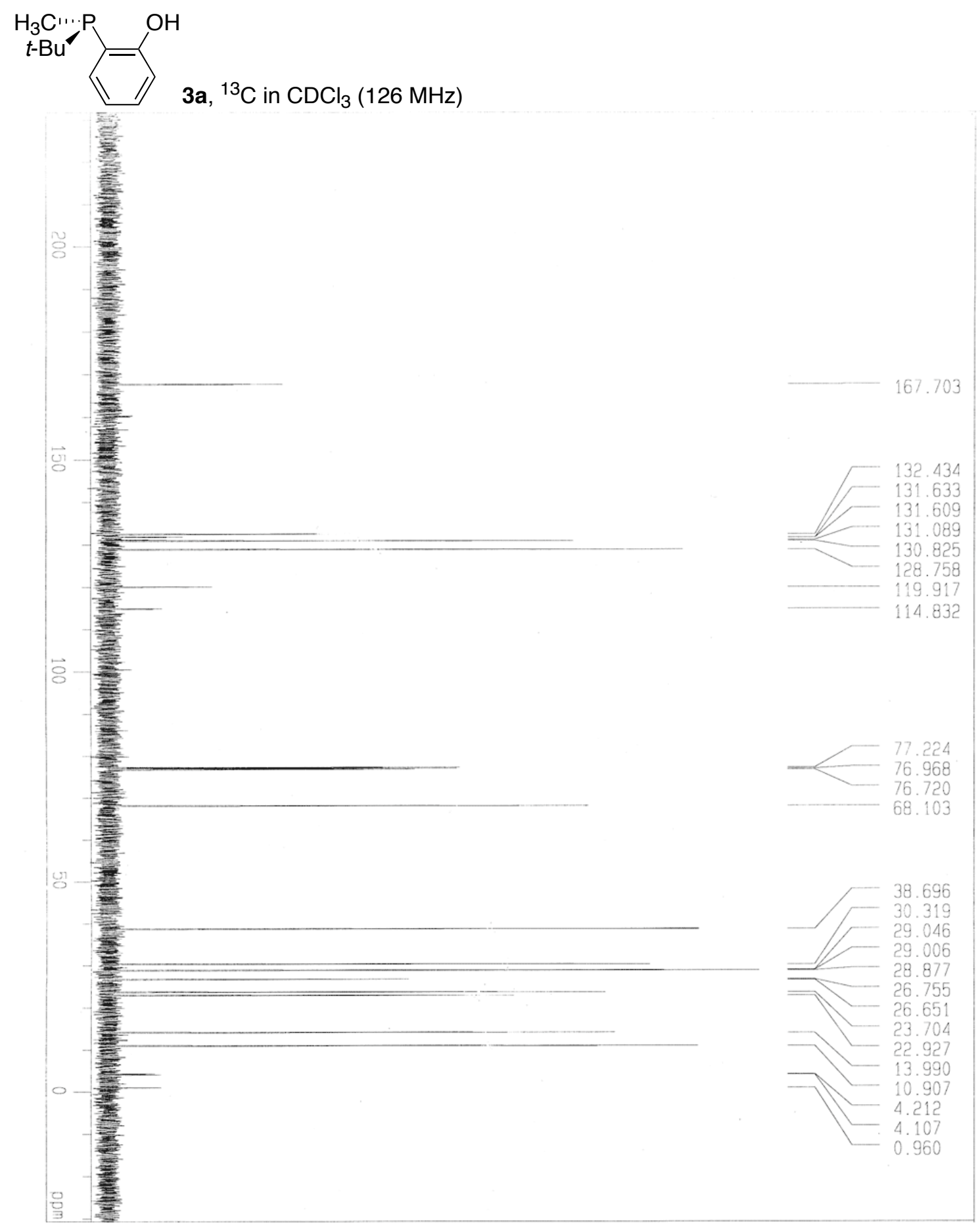




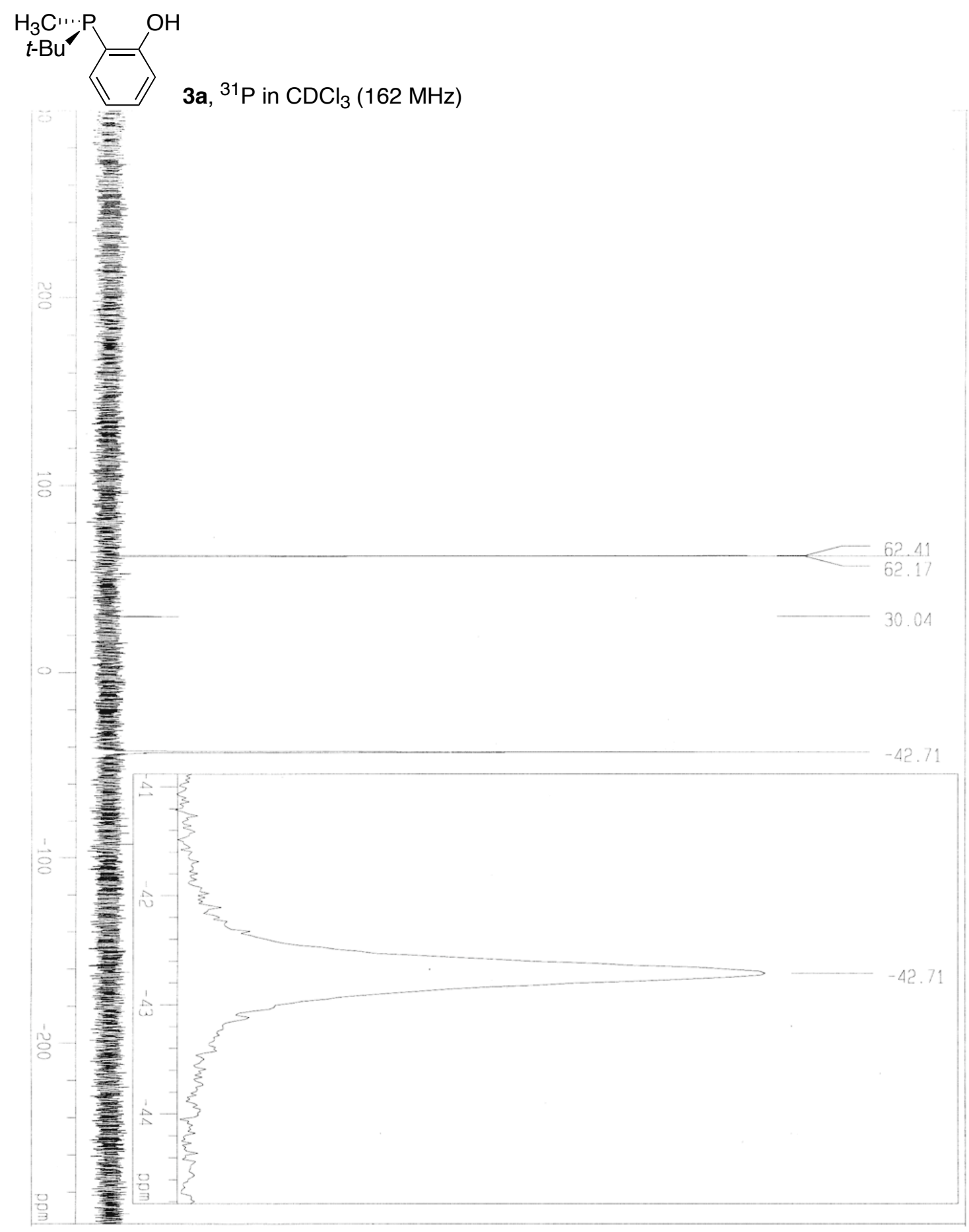




$$
\text { 3b, }{ }^{1} \mathrm{H} \text { in } \mathrm{CDCl}_{3}(500 \mathrm{MHz})
$$

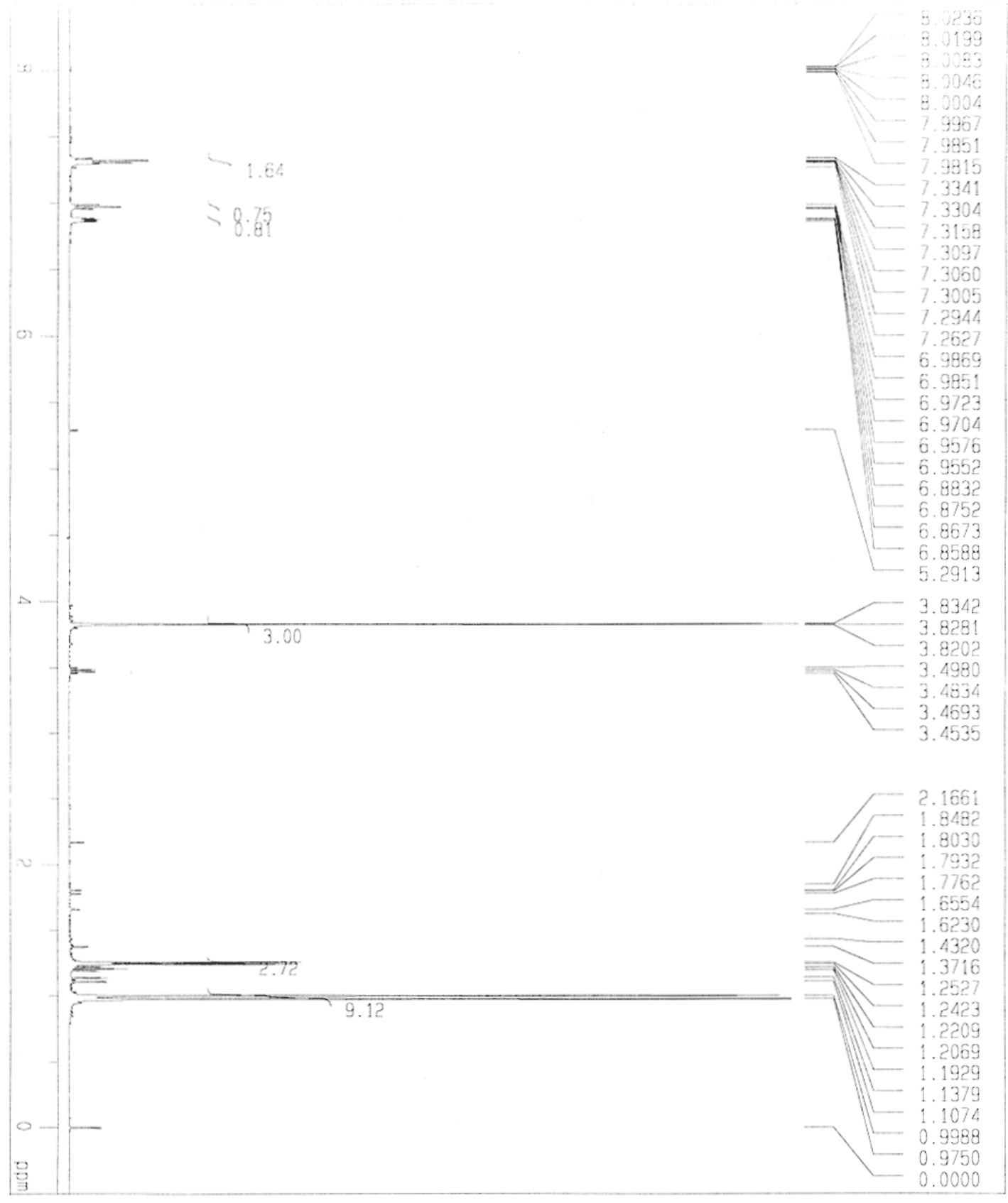




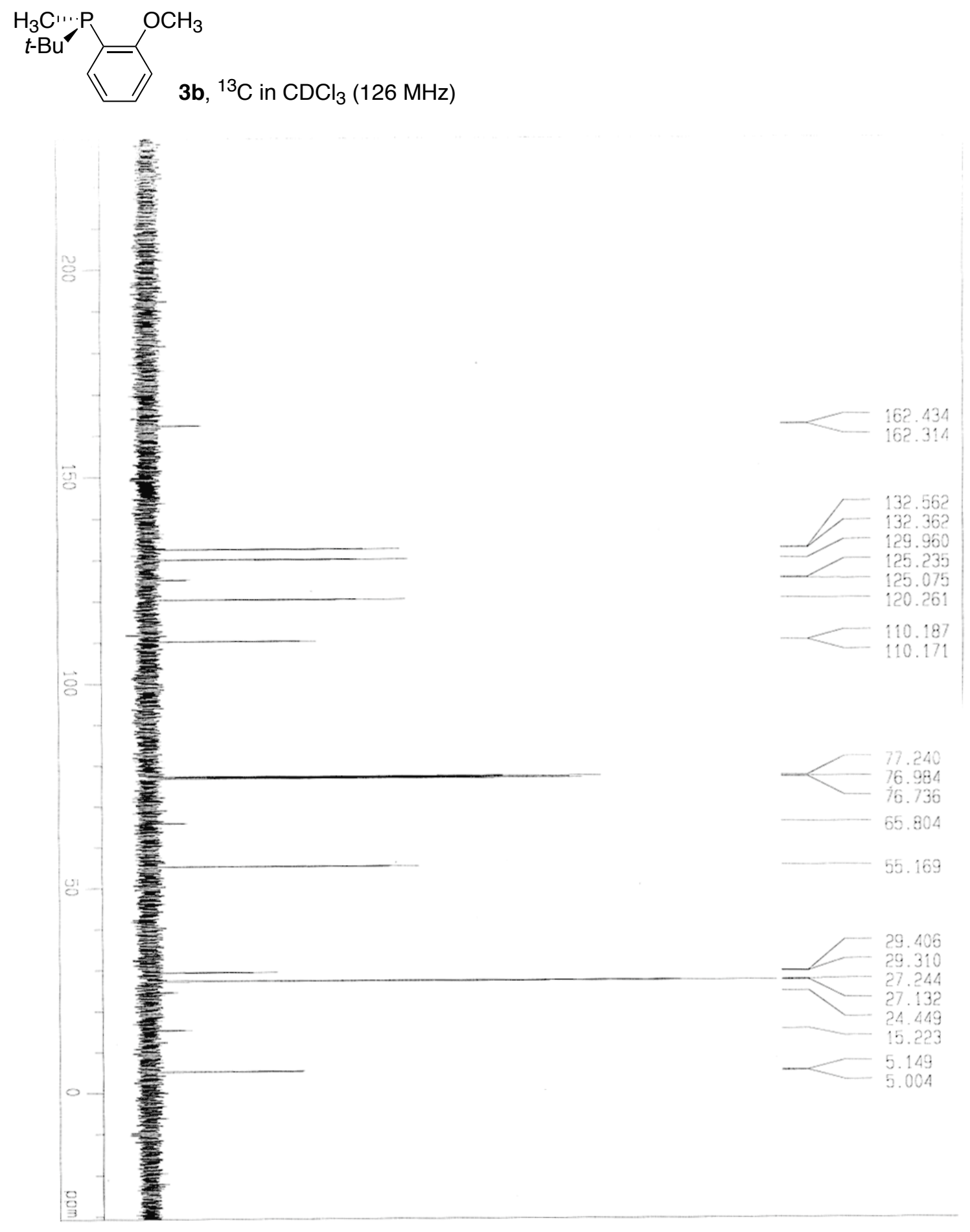

- S20 - 


$$
\underbrace{\mathrm{OCH}_{3}}_{3 \mathbf{b},{ }^{31} \mathrm{P} \text { in } \mathrm{CDCl}_{3}(202 \mathrm{MHz})}
$$

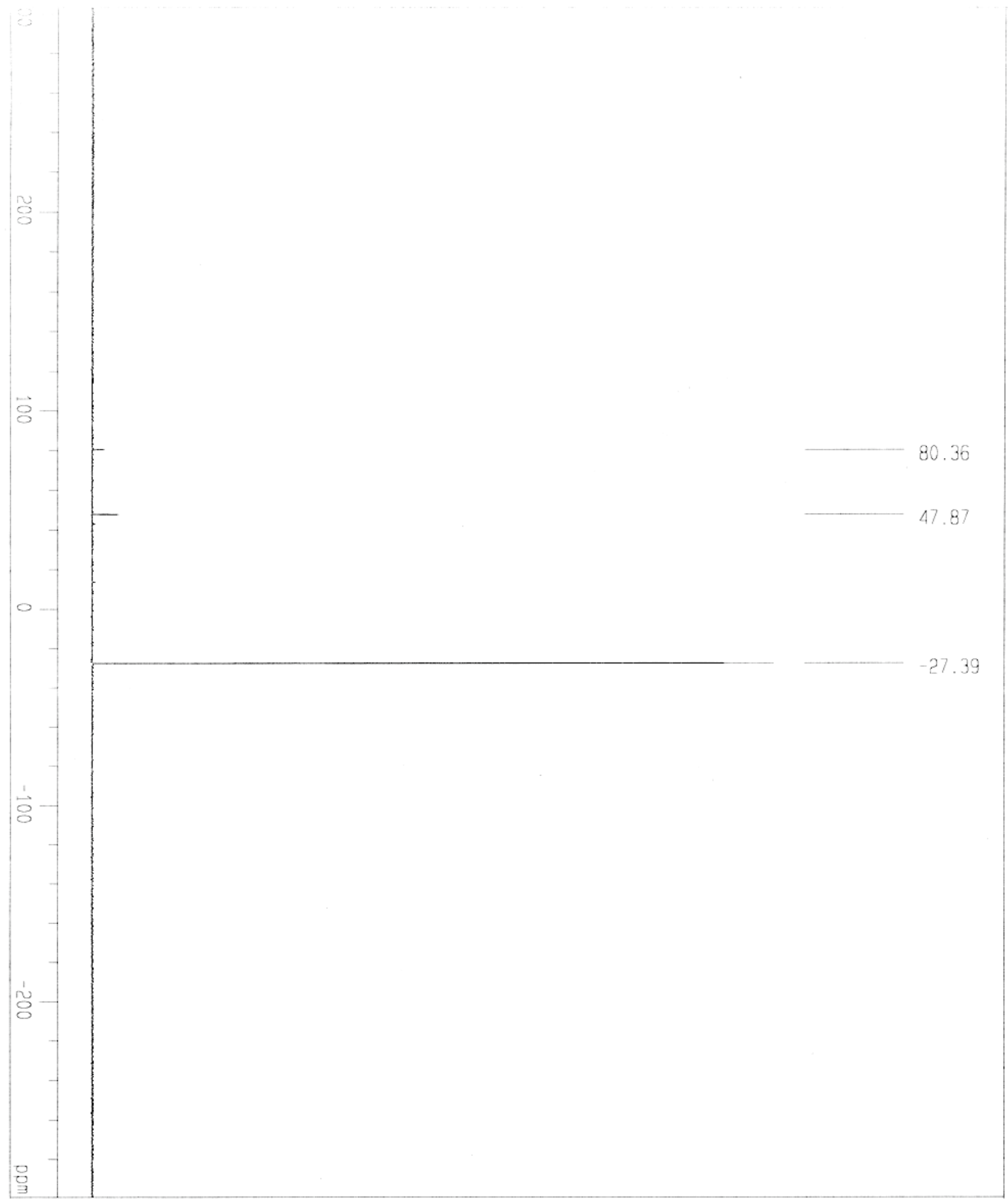




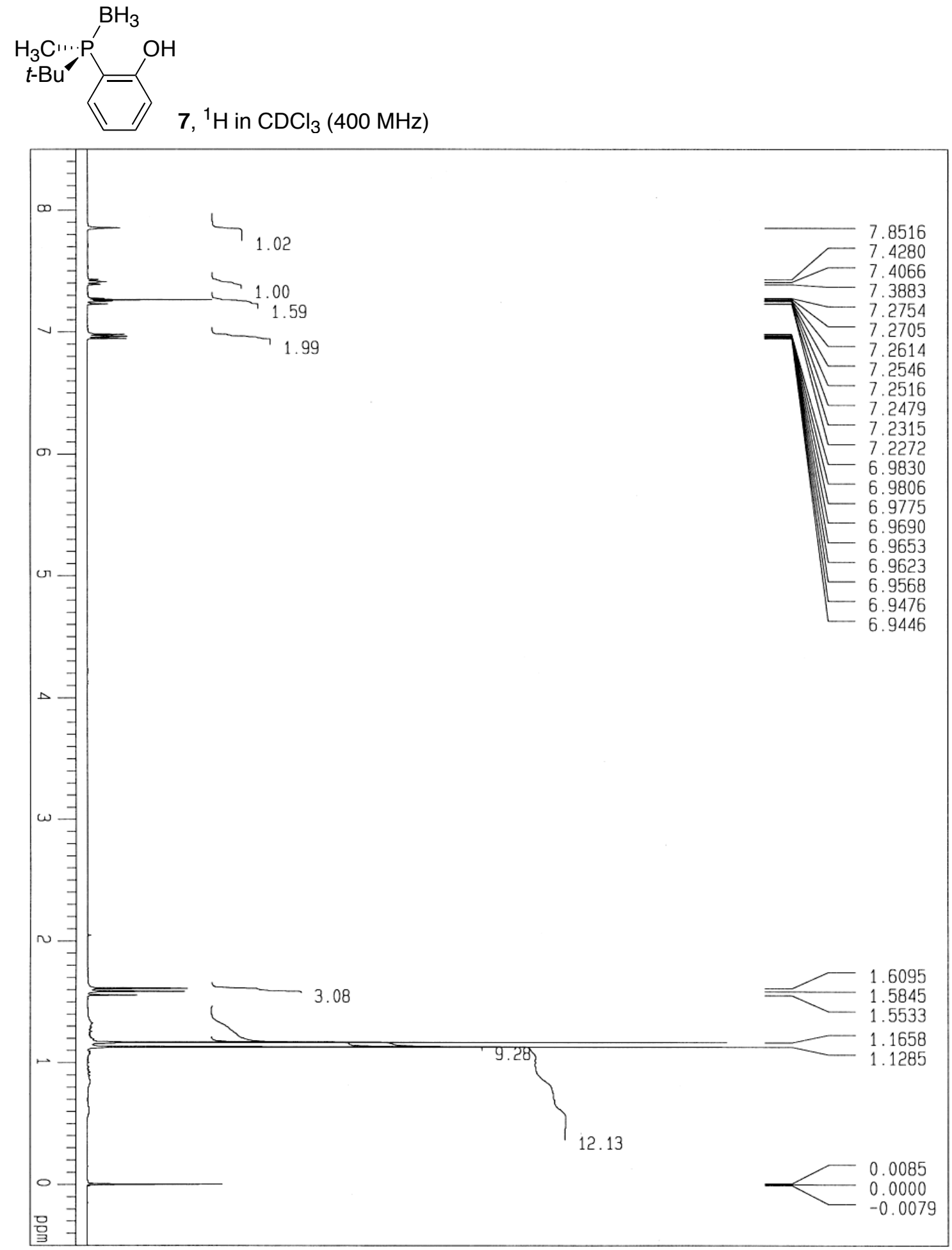



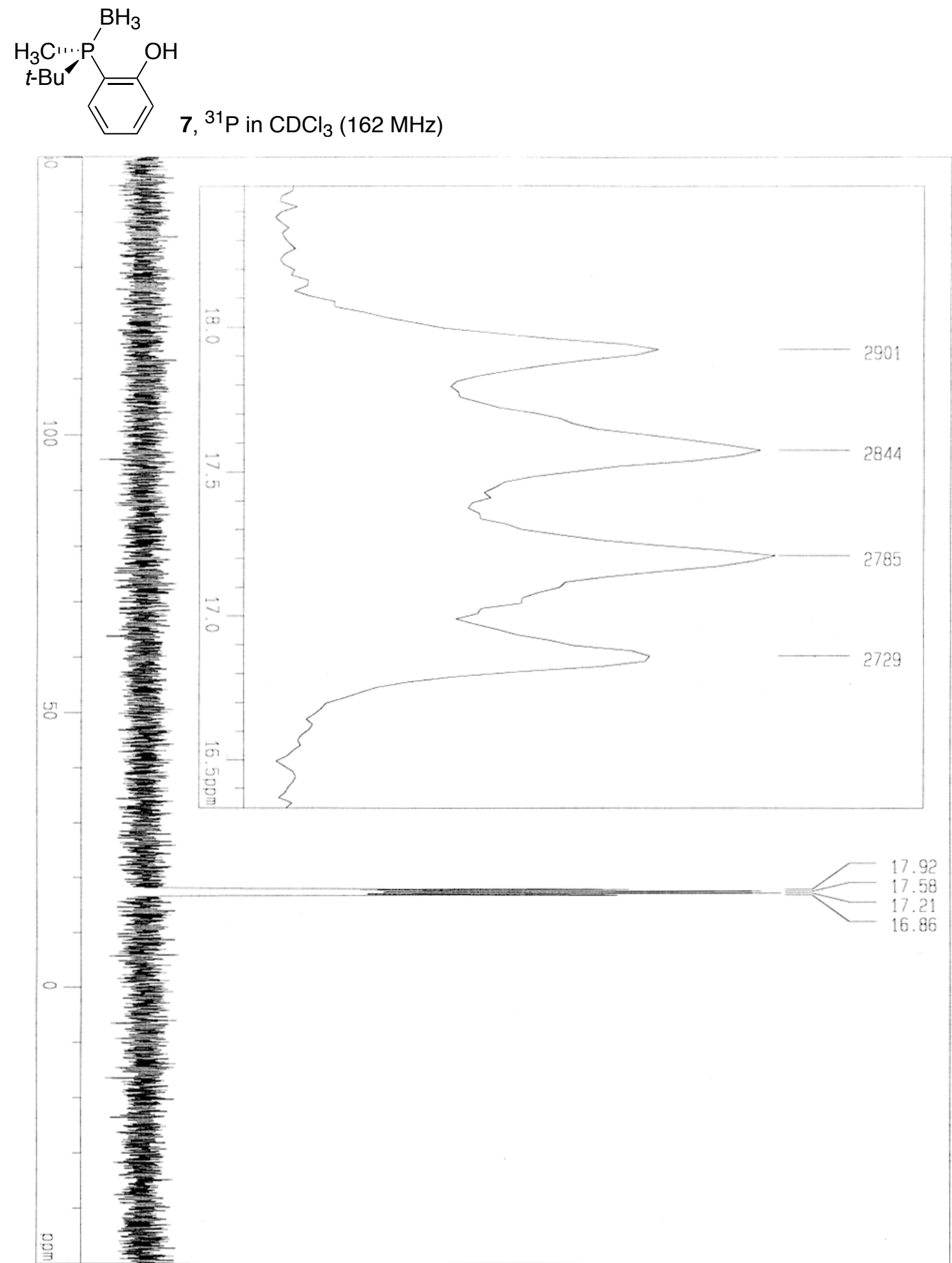


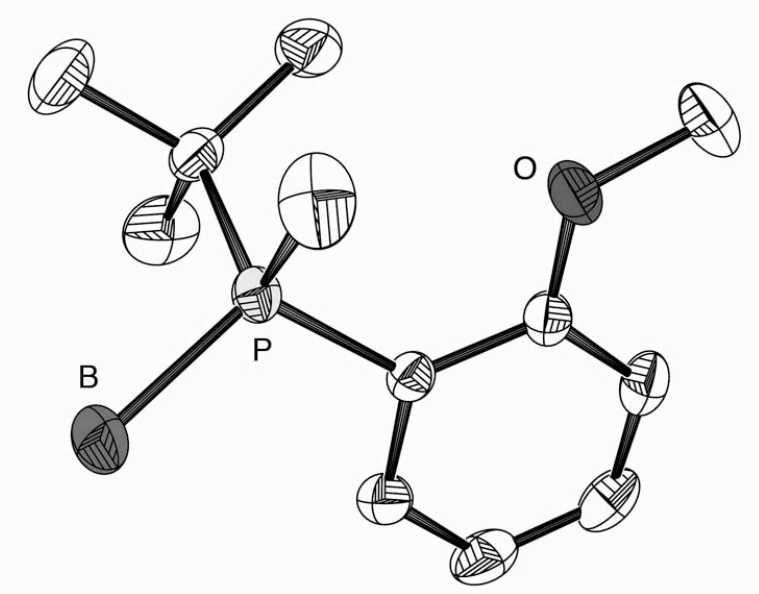

X-ray structure of $\mathbf{2 b}$. All hydrogen atoms were omitted for clarity. 